San Jose State University

SJSU ScholarWorks

Master's Theses

Master's Theses and Graduate Research

Fall 2015

\title{
Synthesis, Structural Characterization And Chromatographic Evaluation Of Silica Hydride-Based Perfluorinated Stationary Phase
}

Harshada Suyog Natekar

San Jose State University

Follow this and additional works at: https://scholarworks.sjsu.edu/etd_theses

\section{Recommended Citation}

Natekar, Harshada Suyog, "Synthesis, Structural Characterization And Chromatographic Evaluation Of Silica Hydride-Based Perfluorinated Stationary Phase" (2015). Master's Theses. 4657.

DOI: https://doi.org/10.31979/etd.2ek3-5erj

https://scholarworks.sjsu.edu/etd_theses/4657

This Thesis is brought to you for free and open access by the Master's Theses and Graduate Research at SJSU ScholarWorks. It has been accepted for inclusion in Master's Theses by an authorized administrator of SJSU ScholarWorks. For more information, please contact scholarworks@sjsu.edu. 

EVALUATION OF SILICA HYDRIDE-BASED PERFLUORINATED STATIONARY PHASE

\author{
A Thesis \\ Presented to \\ The Faculty of the Department of Chemistry \\ San José State University \\ In Partial Fulfillment \\ of the Requirements for the Degree \\ Master of Science
}

By

Harshada Natekar

December 2015 
(C) 2015

Harshada Natekar

ALL RIGHTS RESERVED 
The Designated Thesis Committee Approves the Thesis Titled SYNTHESIS, STRUCTURAL CHARACTERIZATION, AND CHROMATOGRAPHIC EVALUATION OF SILICA HYDRIDE-BASED PERFLUORINATED STATIONARY PHASE

\author{
by Harshada Natekar \\ APPROVED FOR THE DEPARTMENT OF CHEMISTRY \\ SAN JOSE STATE UNIVERSITY
}

December 2015

$\begin{array}{ll}\text { Dr. Joseph J. Pesek } & \text { Department of Chemistry } \\ \text { Dr. Roger Terrill } & \text { Department of Chemistry } \\ \text { Dr. Bradley Stone } & \text { Department of Chemistry }\end{array}$ 


\section{ABSTRACT \\ SYNTHESIS, CHARACTERIZATION, AND EVALUATION OF SILICA HYDRIDE- BASED PERFLUORINATED STATIONARY PHASE}

by Harshada Natekar

Silica hydride based stationary phases have demonstrated unique selectivity and specificity for small sized polar molecules. The weakly hydrophobic nature of silica hydride is thought to be the key reason for its remarkable retention capabilities.

Traditional stationary phases bonded with fluorine moieties have also exhibited enhanced retention of small polar molecules. In this research, a perfluorinated silica hydride stationary phase was synthesized by hydrosilation and silanization procedures. Chromatographic characterization of the newly packed column was carried out by a large number of retention studies. The separation capabilities of the column were evaluated in both the aqueous normal-phase (ANP) and reversed-phase (RP) modes. The perfluorinated silica hydride based column showed remarkable retention ability for RP and ANP modes of separation. Hydrophilic molecules were strongly retained in the ANP mode and hydrophobic molecules were retained in the RP mode. Effect of varying $\mathrm{pH}$ of mobile phase on the retention time was summarized. Use of acetone as non-polar solvent was demonstrated. In the conclusion, silica hydride based perfluorinated stationary phase successfully exhibited dual retention capability for polar and non-polar analytes. 


\section{ACKNOWLEDGEMENTS}

First and foremost, I would like to express my sincere gratitude to my research advisor Dr. J. J. Pesek for accepting me to work in his research group. I would take this opportunity to thank him for his continuous support and guidance throughout my project, for being flexible and providing freedom of experimenting, and sharing his great expertise in the field of chromatographic separations. I would also like to express my deepest thanks to Dr. Maria Matyska-Pesek for training me in instrumental techniques, for instilling laboratory discipline in me, and for being there in the lab whenever I had difficulty in proceeding. Besides my advisor, I am thankful to my committee members Prof. Bradley Stone and Prof. Roger Terrill for their encouragement, insightful questions, and sparing some time from their busy schedules for evaluating my research work. I would like to thank my fellow lab mate and a dear friend Kathleen Talbott for having fruitful and brainstorming discussions, which surely helped me in my research.

Finally, I would like to thank my husband, Suyog, for his unconditional support and tremendous understanding throughout my research work. Also, I'm immensely grateful to the rest of my family back home to India, for their constant encouragement. 


\section{TABLE OF CONTENTS}

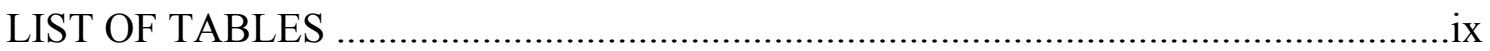

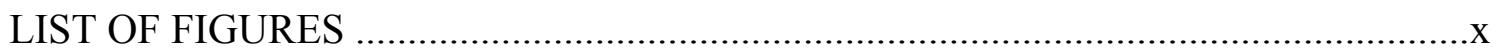

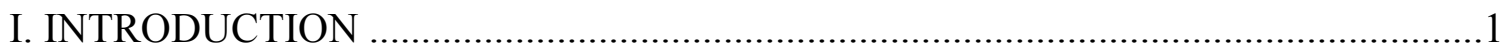

A. Historical Background of Chromatography .....................................

B. High Performance Liquid Chromatography (HPLC) ............................

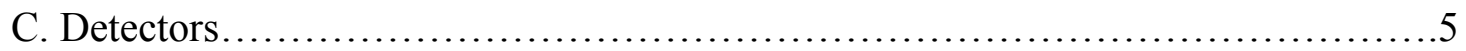

1. UV- Visible Detector...........................................................

2. Mass Spectrometer.................................................... 6

D. Different Modes of Separation in HPLC .................................... 8

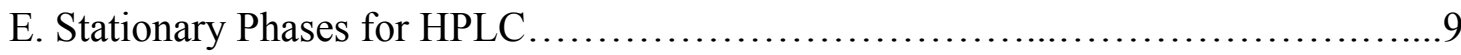

F. Aqueous Normal Phase Chromatography....................................12

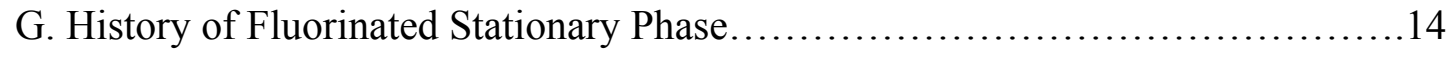

H. Surface Modification of Silica........................................... 15

I. Characterization of Modified Silica Hydride..................................18

1. Diffuse Reflectance Infrared Fourier Transform (DRIFT) ....................18

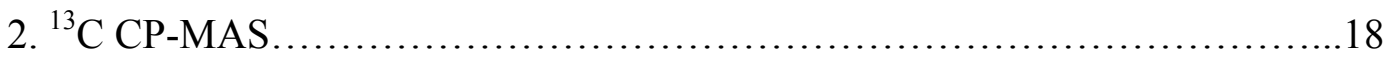

3. Elemental Analysis.................................................... 19

J. Column Evaluation by Variety of Samples...................................20

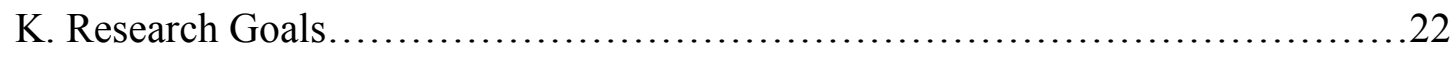

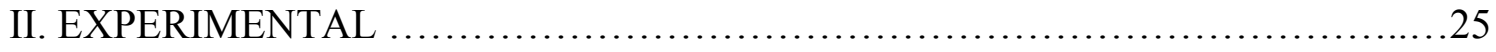

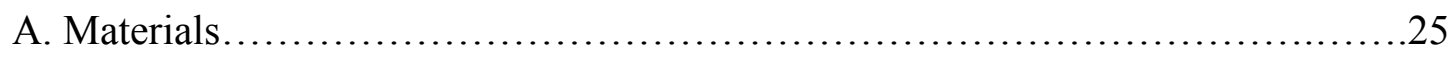




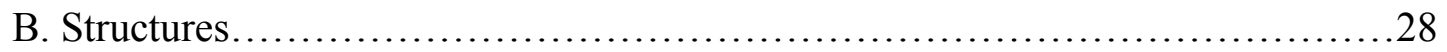

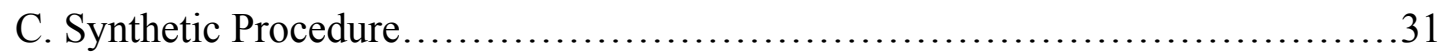

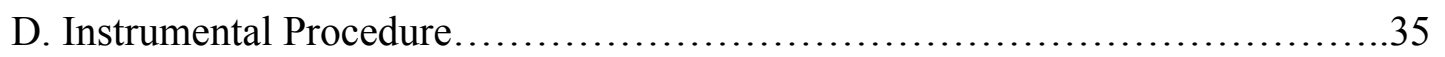

1. Diffuse Reflectance Infrared Fourier Transform (DRIFT) Analysis.............35

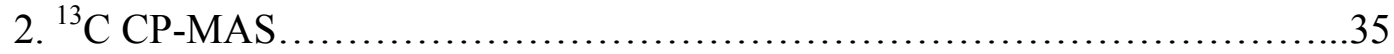

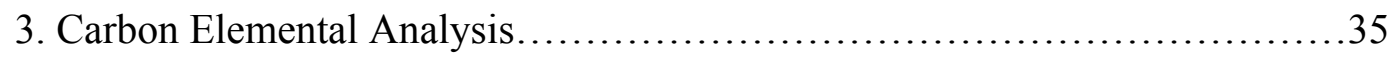

4. High Performance Liquid Chromatography...............................36

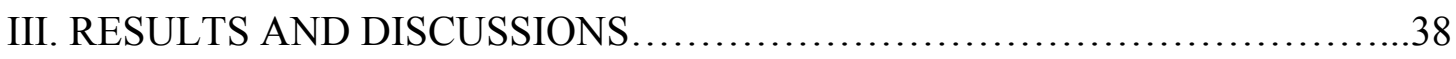

A. DRIFT Spectroscopic Analysis..........................................38

B. Carbon Elemental Analysis............................................42

C. Solid State NMR $-{ }^{13} \mathrm{C}$ CP-MAS ..........................................43

D. Chromatographic Characterization of Stationary Phase.......................45

1. ANP Retention Profile............................................... 45

1.1 Amino Acids...................................................... 45

1.2 Nucleobase related Compounds.....................................48

1.3 ANP Retention of Miscellaneous Compounds.........................52

2. RP Retention Profile...............................................5

3. U-shaped Retention Profile..........................................61

E. pH Studies with Different Buffer Systems................................62

1. Formic Acid Buffer System..........................................62

2. Ammonium Acetate Buffer System.....................................65 
2.1 Compatibility of Ammonium Acetate Buffer With Perfluorinated Silica Hydride-based Stationary Phase ......................................66

2.2 Varying Concentration of Ammonium Acetate Buffer...................69

F. Comparison of Acetone and Acetonitrile as Solvent B.......................72

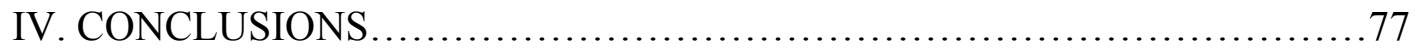

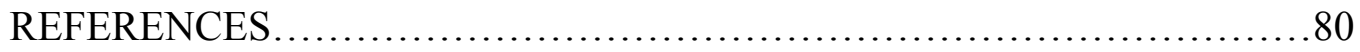




\section{LIST OF TABLES}

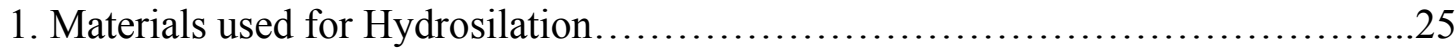

2. Chemicals required for Mobile Phase Preparation..................................26

3.1. Nucleobases, Nucleosides, and Nucleotides..................................26

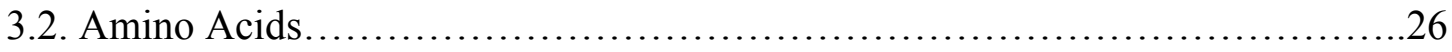

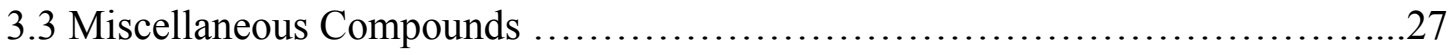

4. Samples Analyzed for RP Retention of Stationary Phase........................27

5. Surface Coverage of Bonded Organic Moiety ...................................44

6. Retention Time for Amino Acids .............................................47

7. Retention Time for Adenosine, Cytosine, and Guanine..........................49

8. Retention Time for Thymine and Uracil......................................49

9. Retention Time for Creatinine and Creatine Hydrate.............................54

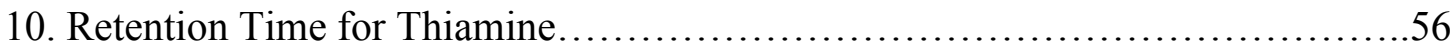

11. Retention Time for Fluorene, Pyrene, Naphthalene, and Phenanthrene............59

12. $\mathrm{pH}$ values for Different Concentrations of Formic Acids.........................63

13. Retention Time (Minutes) at Different Concentrations of Formic Acid..........66

14. Retention Times for Polar Solutes with Ammonium Acetate Buffer.............67

15. $\mathrm{pH}$ values for Different Concentrations of Ammonium Acetate Buffer...........70

16. Retention Time at Different Concentrations of Ammonium Acetate Buffer.......70

17. Retention Factors for L-Phenylalanine...................................... 74

18. Retention Factors for L-Tryptophan.................................................................74 


\section{LIST OF FIGURES}

1. Schematic Representation of HPLC System..................................

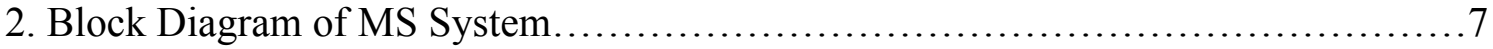

3. Geminal and Vicinal Forms of Silanol Groups................................ 10

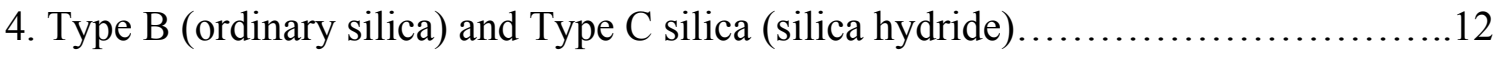

5. Water association with Type $\mathrm{B}$ and Type $\mathrm{C}$ silica..................................

6. Schematic representation of silanization/hydrosilation procedure..................17

7. Generic plot for retention profile of silica hydride based stationary $\ldots \ldots \ldots \ldots \ldots \ldots . .24$

8. Experimental set up for hydrosilation reaction............................... 33

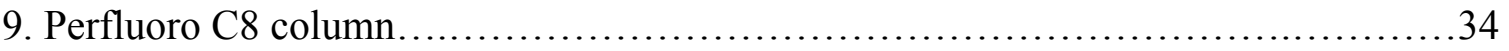

10. HP/Agilent 1050 Series HPLC-UV Instrument ............................ 36

11. Agilent 1100 series (TOF) LC-MS ...................................... 37

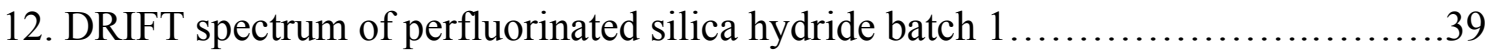

13. DRIFT spectrum of perfluorinated silica hydride batch $2 \ldots \ldots \ldots \ldots \ldots \ldots \ldots \ldots \ldots$

14. Overlap of DRIFT spectra of perfluorinated silica hydride batch 1 and batch $2 \ldots . .41$

15. Bonding between Si and perfluorinated moiety...........................44

16. ${ }^{13} \mathrm{C}$ CP-MAS Spectrum of perfluoro silica hydride...........................44

17. ANP Retention of Amino Acids...........................................47

18. ANP Retention of Adenosine, Cytosine, and Guanine........................51

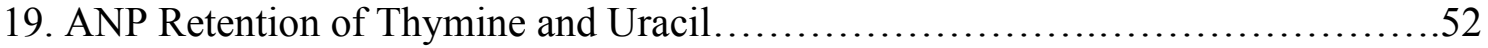

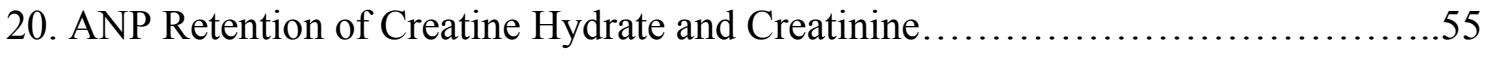


21. ANP Retention of Thiamine.

22. RP Retention of Pyrene, Naphthalene, Fluorine, and Phenanthrene..............60

23. U-shaped Retention Profile for Perfluorinated Silica Hydride Stationary Phase....63

24. (A-E). Retention Time as a Function of Concentration of Formic Acid ............64

25. Retention of Polar Solutes with Ammonium Acetate Buffer.......................68

26. (A-D). Retention Time as a Function of Concentration of Ammonium Acetate.....71

27. Retention Factor as a Function of \% B for L-phenylalanine......................75

28. Retention Factor as a Function of \% B for L-tryptophan.......................76 


\section{INTRODUCTION}

\section{A. Historical Background of Chromatography}

Chromatography is a powerful separation technique that finds applications in diverse branches of science. It involves separation and identification of closely related compounds in a mixture. It is highly efficient and selective compared to other traditional separation methods like precipitation or distillation. Chromatography plays a crucial role in the separation of complex mixtures.

The word chromatography originates from two Greek words chroma and graphein. Chroma means color and graphein means to write; hence chromatography literally means writing with colors. Discovery of chromatography is attributed to the Russian botanist Mikhail Tswett. In the year 1903, he separated plant pigments in a glass column packed with calcium carbonate. Separation of plant pigments like chlorophyll and xanthophyll produced colored bands and therefore he coined the term chromatography. With the tremendous advancements in the technique over the century, 'color' no longer plays a role in the identification process. However, the principles of separation still apply [1].

Chromatography has two main components: the stationary phase and the mobile phase. As the names suggest, the stationary phase remains still and the mobile phase runs through the stationary phase. Analyte molecules also travel through the stationary phase along with the mobile phase. Different types of physical and chemical interactions between the analytes in the stationary phase and the mobile phase, govern their affinities toward each other. The analytes, which are strongly retained on the stationary phase, move slowly with the flow of the mobile phase. Analytes that are weakly held on the 
stationary phase move faster with the mobile phase and elute first. Based on their migration velocities, components of the mixture are eluted at different times and hence they get separated [2].

Chromatography is an umbrella term that encompasses different modes of chromatographic separations. Classification of the chromatographic techniques can be done based on various parameters, including the type of stationary phase and mobile phase and the mechanism of separation. They can be broadly classified into planar chromatography and column chromatography. In planar chromatography, a thin plate or a paper supports the stationary phase, and the mobile phase runs through it by capillary action or gravitational pull. The most common technique is known as column chromatography, in which the stationary phase is packed in a narrow column and the mobile phase is forced through the column under pressure. Based on the physical nature of the mobile phase, column chromatography is further classified into two main categories, gas chromatography and liquid chromatography. In both of these types, the stationary phase is solid or liquid supported on solid particles. The terms "liquid chromatography" and "gas chromatography" indicate the mobile phase being liquid and solid, respectively. Gas chromatography is mainly used for volatile analytes that can be vaporized without decomposition [2,3].

Liquid chromatography is the most commonly used technique of all the analytical separation methods. Widespread use of liquid chromatography is mainly due to its sensitivity, suitability for separation of the broad range of analytes, adaptability to different experimental conditions, and applicability to the substances that are most 
important for industries, like health care, pharmaceuticals, pesticides, food production, basic chemicals and hydrocarbons, and biotechnology.

\section{B. High Performance Liquid Chromatography (HPLC)}

High performance liquid chromatography (HPLC) is a highly efficient and advanced version of liquid chromatography. During the early 1960s, it was referred to as high "pressure" liquid chromatography because, technically, high pressure is used to push the mobile phase through the column. In the late 1970s, because of advancements in the column material, efficient pressure pumps and the overall technical development in the instrumentation led to enhanced performance, hence the term "high pressure" was changed to "high performance" liquid chromatography. Typically, a sample mixture is dissolved in a solvent and flushed through the column under high pressure, along with the mobile phase. The stationary phase, which is packed in a column, resolves the sample mixture into its individual constituents. Some of the primary components of the modern HPLC instrumentation include the mobile phase reservoir, the solvent treatment system, the pumping system, the sample injection system, the column for separation, the detector, and the data processor [4].

Typically, the instrument is provided with four solvent reservoirs, made up of glass or plastic. The degasser is employed before the solvent injection system. It removes any air or dissolved gas from the solvent. Dissolved gases might cause irreproducible flow rates and band broadening. Air bubbles and dust ruin the stationary phase and can cause interference in the detection system. The degasser can either work by helium purging or vacuum degassing. The pressure pumps that are used in current day HPLC instruments 
generally produce a maximum pressure up to $6000 \mathrm{psi}$ ( $400 \mathrm{bar}$ ) and flow rates ranging from 0.1 to $10 \mathrm{~mL} / \mathrm{min}$. The sample injection system can be manually operated or automated. Manual sample injection includes a sampling loop, which can hold sample sizes from 1 to $50 \mu \mathrm{L}$. The sample is manually injected in the loop with the help of a syringe and a single switch valve. The manual injection method results in human error in sample size. More recently, auto samplers/auto injectors have become popular, mainly because of the accuracy of the sample volume. The auto injector also has a sampling loop and a syringe. The injector takes the sample from a specified vial on the sample carousel and injects the sample into the column [5].

The column is referred to as the "heart of chromatography" because it is the column where the separation takes place. HPLC columns are made of stainless steel and packed with a silica-based stationary phase. In general, analytical columns are 5 to $25 \mathrm{~cm}$ long, 3 to $5 \mathrm{~mm}$ in diameter, and 3 to $5 \mu \mathrm{m}$ is the particle size of the packed material. Highly skilled workers and specialized equipment is required for column packing. Columns are generally bought from professional manufacturers. They are durable and can last a long time unless they are treated with harsh solvents or get contaminated with dust or sample impurities.

The mobile phase and analytes enter a suitable detector once they are eluted from the column. The detector produces an electrical signal proportional to the amount of sample, and a chromatogram is produced. The chromatogram shows the peaks for specific analytes in the order in which analytes are eluted. An analyte that is not retained on the column for a long time elutes first and the one that is retained for the longest time elutes 
last. Different types of suitable detectors are used for efficient detection of the analytes. Some of the commonly used detectors are refractive index detector, diode array, UV-Vis and mass spectrometer. Figure 1 represents the schematic arrangement of an HPLC instrumentation [6].

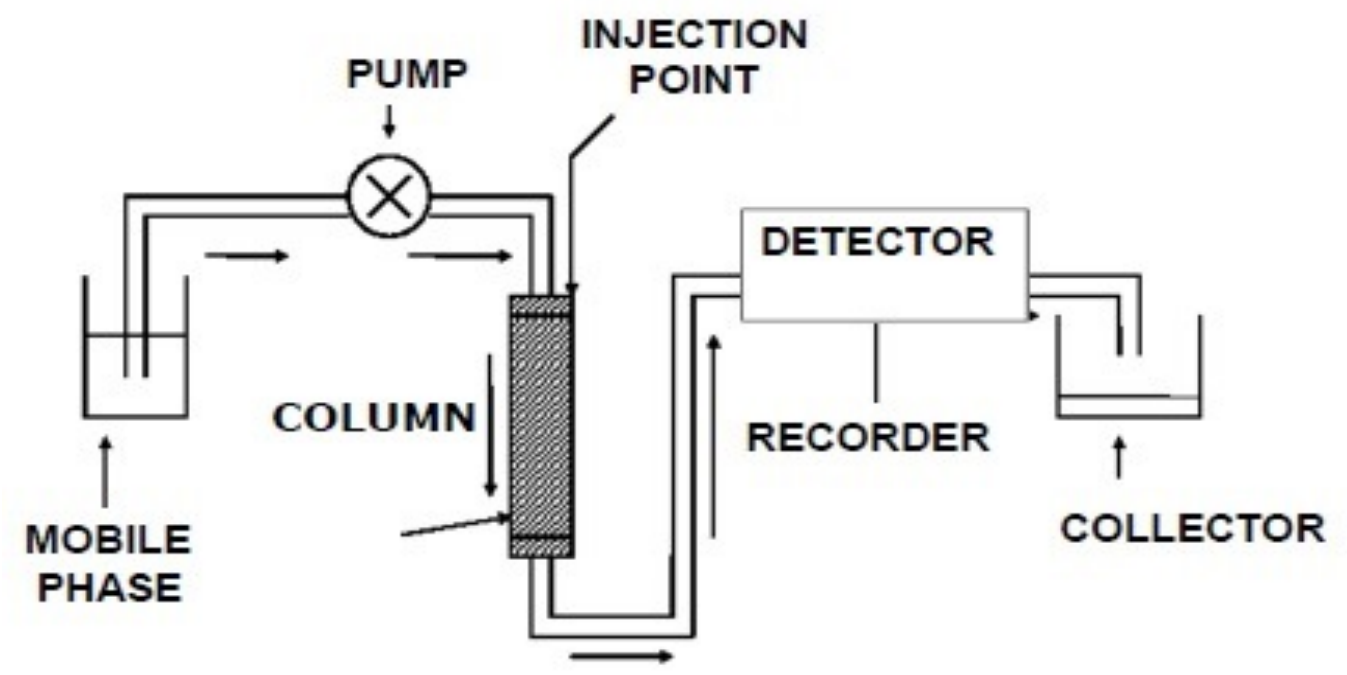

Figure 1: Schematic Representation of HPLC System [7].

\section{Detectors}

The traditional analytical detectors can be incorporated into an HPLC system for the detection of a target analyte from the eluent. The ideal detector should have adequate sensitivity, it should have good stability and reproducibility, and it should be nondestructive. The detector should have minimal internal volume in order to reduce the zone broadening [2]. Solute property detectors are more commonly used for modern 
HPLC applications. Mass spectrometers (MS) are becoming more common in modern analytical laboratories.

\section{UV-Visible Detector}

Many compounds in nature can absorb light in the ultraviolet-visible region (200 to $800 \mathrm{~nm}$ ) of the spectrum. Absorption of light by a chromophore is governed by the BeerLambert law, which states that "the amount of energy absorbed or transmitted by a solution is proportional to the solution's molar absorptivity and the concentration of solute". In other words, the amount of light absorbed by a particular solute at a specific wavelength is directly proportional to its concentration. A UV-Vis detector works on the principle of the Beer-Lambart law. It is the simplest and most commonly used detector for HPLC. Different filters or a monochromator provide a specific wavelength selection. Advanced detectors allow scanning of multiple wavelengths at the same time. This detector is highly sensitive, reliable, versatile, and easy to operate. However, it must be noted that the analyte should absorb in the UV-Vis range of the light to make use of this detector $[1,2]$.

\section{Mass spectrometer}

Mass spectrometry (MS) is the most versatile and selective detection technique. Identification and quantification of the target analytes are based on the measurement of their mass to charge ratios $(\mathrm{m} / \mathrm{z})$. Generally, there are three main components of any mass spectrometer: the ion source, the mass analyzer, and the ion detector [8]. 
The combination of liquid chromatography and mass spectrometry (LC/MS) forms a powerful technique of separation and identification of analytes. Figure 2 shows a block diagram of the typical MS system.

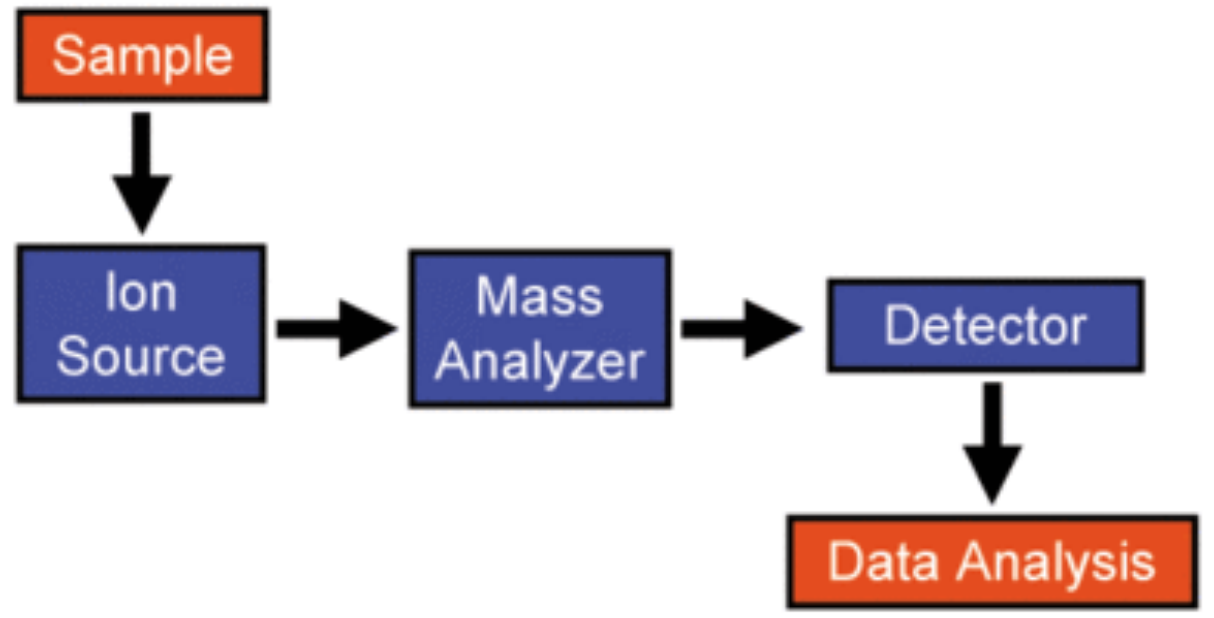

Figure 2: Block Diagram of MS System

In the LC/MS system, compounds of interest, once separated and eluted from LC, are introduced into an ion source. These compounds are converted into a gaseous ionic species, and the majority of the eluent goes into the waste. This is a crucial step to convert the liquid compounds into the gas phase ions. It is important to get rid of the solvent while maintaining a vacuum in the mass spectrometer; hence, choosing an appropriate LC interface is important. Some of the popular ion sources are atmospheric pressure chemical ionization (APCI) and electrospray ionization (ESI). Both these techniques involve soft ionization of the target molecules. 
ESI is the most commonly used ion source for biomolecules. It operates at atmospheric pressure. A small capillary introduces eluent into the ion source setup. The high voltage is applied at the tip of the capillary; as a result, the solution forms a Taylor cone at the tip of the capillary. A high potential difference is maintained across the electrodes to create a steady spray of droplets, which are reduced by solvent evaporation, leaving gaseous analyte behind [8].

The gaseous ions formed in the ion source are then pumped into the next component of the MS system, which is the mass analyzer. Popular mass analyzers include ion trap, magnetic sector, quadruple, and time of flight (TOF). The mass analyzer can selectively isolate desired species with a specific $\mathrm{m} / \mathrm{z}$ or it can scan all the $\mathrm{m} / \mathrm{z}$ ratios in samples. In the quadruple mass analyzer, a combination of AC and DC fields allow only ions with a specific $\mathrm{m} / \mathrm{z}$ to pass through to the detector. The time of flight mass analyzer makes use of the variable acceleration of the charged ions based on their $\mathrm{m} / \mathrm{z}$ ratio $[3,8]$.

\section{Different Modes of Separation in HPLC}

Based on the polarity of the stationary phase and the mobile phase, there are two main modes of separation in HPLC: the normal-phase (NP) and the reverse-phase (RP) modes. In the normal-phase mode, the stationary phase is polar and the mobile phase is nonpolar. In the reverse-phase mode, the stationary phase is nonpolar and the mobile phase is polar. The reverse-phase mode is most commonly used and is capable of retaining nonpolar compounds.

In principle, polar compounds can be retained on NP type stationary phases. But in practice, many biomolecules and pharmaceutical samples have multiple polar sites, and it 
becomes impossible to have a separation method based on just RP or NP modes. Since these polar compounds are an important class of molecules, and it is absolutely essential to have an effective protocol for retention of polar moieties. There are different strategies to enhance their retention, like using extreme $\mathrm{pH}$, the use of ion pairing reagents, and temperature variation. But, none of these techniques are efficient enough for most applications, so different HPLC techniques have been developed for separation of small polar molecules [1,2].

Hydrophilic interaction liquid chromatography (HILIC) is one of the common methods used for the separation of polar compounds. HILIC has a polar stationary phase with the silanol rich surface. It may be considered as a type of normal-phase mode chromatography. The mobile phase has a high amount of nonpolar solvent. The separation mechanism is believed to be partitioning between the polar aqueous layer on the surface of the stationary phase and the highly nonpolar mobile phase. It is extensively used for separation of the biomolecules and the small polar compounds [6].

\section{E. Stationary phases for HPLC}

Silica is the preferred backbone for HPLC stationary phases. The chemical composition of the silica consists of $\mathrm{SiO}_{2} \cdot \mathrm{H}_{2} \mathrm{O}$. Its structure involves the backbone network of siloxane bonds ( $\mathrm{Si}-\mathrm{O}-\mathrm{Si})$. The silanol $(\mathrm{Si}-\mathrm{OH})$ groups cover the surface of the silica, which could be in the geminal or the vicinal forms (Figure 3). 


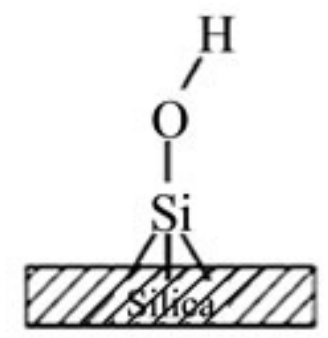

Isolated Silanol

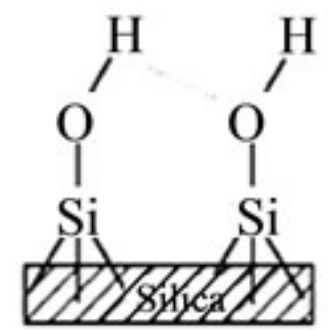

Vicinal Silanol
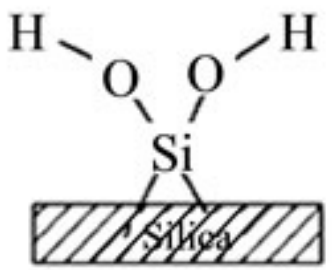

Geminal Silanol

Figure 3: Geminal and Vicinal Forms of Silanol Groups [10]

The excellent physical properties of the silica make it the most suitable choice for stationary phase applications. Silica particles can withstand high pressure and a constant flow of liquids. In HPLC systems, high pressure is applied to flush the mobile phase through the stationary phase. Silica particles also have high mechanical strength and rigidity; hence, it can be packed into the column very well. It also has advantages of being stable in various physical forms. Silica particles can be manufactured in a broad range of sizes. It is relatively inert and can be easily modified on the surface. This allows the bonding of a desired organic moiety on the surface of silica matrix. Another most important property of silica is that it provides a very high surface area. This makes it possible to have small lengths for columns, which provide sufficient surface area for analyte - stationary phase interactions [9].

\section{Type B silica}

Type B silica, also known as ordinary silica, has silanol groups $(\mathrm{Si}-\mathrm{OH})$ on the surface. This is the most commonly used backbone for HPLC stationary phases. The Type B silica is modified with organic moieties such as, diols, undecenoic acids, on the 
surface. Some of the commercially available and commonly used phases are C8, C18, amino groups, and diols bonded Type B stationary phases.

Although Type B silica is most widely used, it has significant limitations. For example, hydrophilic/polar compounds are poorly retained on reverse phase mode with modified type B stationary phases. There is instability at high $\mathrm{pH}$. It cannot retain bases at low $\mathrm{pH}$ and acids at high $\mathrm{pH}$. There could be on-column degradation of analytes because of the adsorbed layer of water on the surface. Often, there are long run times and long equilibrium times between successive gradients.

2. Type C silica

Pesek et al introduced a new type of stationary phase known as Type C silica, which is believed to be capable of overcoming some shortcomings of type B/ordinary silica. Type C silica has a similar silica backbone as that of type B; however, it has silica hydride (SiH) groups on the surface. Replacing the $\mathrm{Si}-\mathrm{OH}$ of ordinary silica by Si-H dramatically changes the surface behavior; hence, there are resulting changes in the separation mechanism and the retention patterns of analytes. The basic difference is silica hydride is weakly hydrophobic in nature. In the case of silica hydride, there is a weak adsorption of water as opposed to strong water adsorption as in the silanol groups. It is suspected that silica hydride does not form an adsorbed layer of water on the surface; hence, the separation mechanism should be different from Type B silica [12]. Figure 4 illustrates Type B and Type C silica. Figure 5 shows the strong and weak association of water molecules with Type B and Type C silica, respectively. 
Ordinary Silica

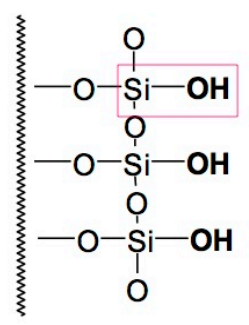

Silica Hydride

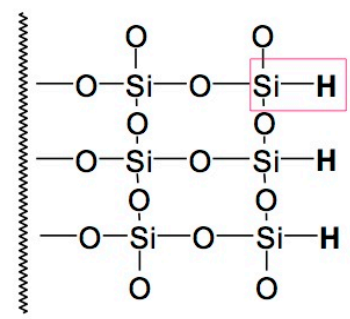

Figure 4: Type B (ordinary silica) and Type C silica (silica hydride) [11].

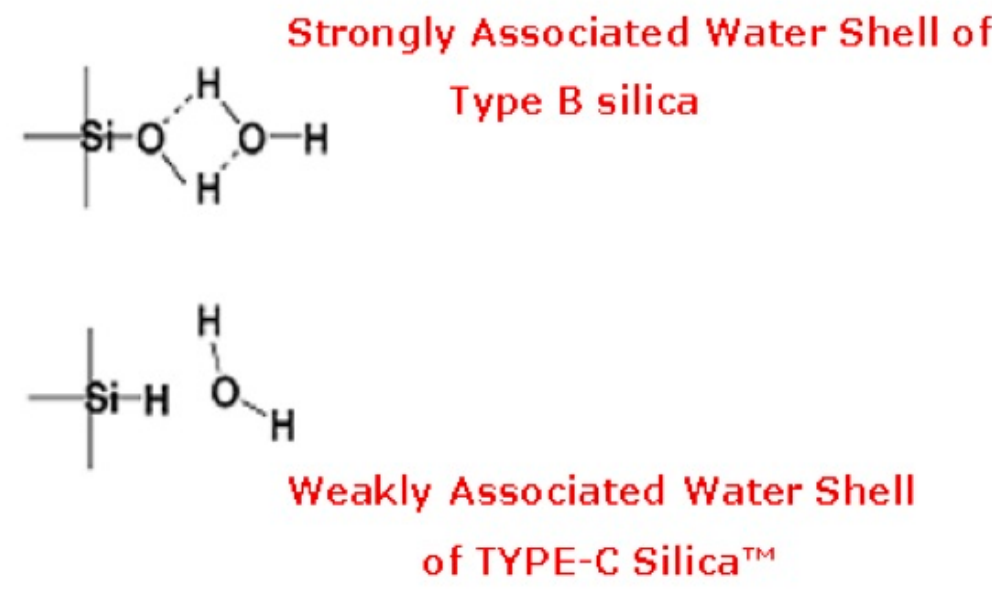

Figure 5: Water Association with Type B and Type C silica [11].

\section{F. Aqueous Normal Phase Chromatography (ANP)}

Aqueous normal phase (ANP) chromatography involves silica hydride-based stationary phases. The mobile phase is a mixture of polar and nonpolar solvents. ANP was introduced by Pesek et al [30]. Research has demonstrated many remarkable characteristics of silica hydride-based ANP retention in HPLC. By far, it is the most versatile method of separation [15]. The polarity of the mobile phase can be changed 
easily by varying the composition of the mobile phase. The ANP mode is capable of retention of polar as well as nonpolar analytes with similar efficiency. When there is an increase in the percent of polar solvent in the mobile phase, there is an increase in retention of nonpolar analytes; conversely and uniquely for $\mathrm{SiH}$ phases, when the percentage of nonpolar solvent increases, there is also an increase in retention of the polar compounds. This dual retention capability of the silica hydride based column is unique. A U-shaped retention profile is being demonstrated by this type of stationary phase, which results in retention of polar and nonpolar compounds [13].

The retention mechanism that is involved in ANP is thought to be different from HILIC. As mentioned earlier, converting Si-OH to Si-H alters the surface chemistry of the stationary phase. This change in surface composition should involve a different retention mechanism for ANP. It could involve more than one complex interaction, like electrostatic forces, adsorption, and ionic affinities [14]. The exact mechanism of ANP retention has not been fully established.

It is important to outline some of the key differences in the HILIC and ANP techniques. The nature of the stationary phase in case of HILIC is highly polar whereas, in ANP, it is weakly hydrophobic. The silica hydride-based stationary phase does not absorb a layer of water as in the case of HILIC stationary phases [15], which forms the hydration shell on the surface. Although HILIC is useful for retention of polar compounds, it does not have the dual retention ability [15]; therefore, if the sample is a mixture of both polar and nonpolar analytes, then distinct methods of RP and HILIC need to be used. Different methods of RP and HILIC will involve different stationary phases and chromatographic 
conditions. ANP is capable of retaining solutes with largely different polarities. Because the silica hydride is highly stable, a wide range of binary solvents can be used.

\section{G. History of Fluorinated Phases}

Fluorocarbons have demonstrated many desirable properties for their use as HPLC stationary phases. Fluorinated alkyl and phenyl bonded stationary phases have selective retention profiles, which are different from traditional C8 or C18 types of retention [16]. Fluorinated columns have been useful for those separations for which traditional C8 or C18 columns are not better options. Fluorinated stationary phases have been complementary to the C8/C18 RP mode of separation.

The Galan group published the very first article in 1980 that described the preparation of a fluorinated stationary phase [17]. They also discussed application of fluorinated phases for separation of fluorinated moieties from non-fluorinated compounds. Xindu and Carr published additional interesting research in 1983. They described the separation of proteins on $\mathrm{C}_{8} \mathrm{~F}_{17}$. They suggested use of a high concentration of organic solvent in the mobile phase instead of ion pairing agent [18]. Replacing $\mathrm{C}-\mathrm{H}$ in olefin chains by a C-F functionality could introduce the dipole character that may enhance retention of poplar compounds $[16,19]$.

Zhang reported that the fluorinated phase has a U-shaped retention profile for small polar particularly basic compounds [20]. At the left part of the U-shaped profile, retention of polar solute decreases with an increase in concentration of the organic solvent. After passing a certain low point, there is an increase in the retention time for polar basic compounds with an increase in concentration of organic solvent in the mobile phase. 
Fluorinated phases have shown higher selectivity toward fluorinated species over nonfluorinated species. Fluorinated phases are particularly useful when sample consist of a mixture of fluorinated and non-fluorinated compounds. Various separation mechanisms have been proposed: pi-pi interaction for phenyl-based solutes, charge transfer, and ionic interaction for polar solutes.

Mass spectrometry is becoming increasingly popular and is one of the reliable techniques of detection. ANP mode of separation is useful for fluorinated phases because it involves use of high percent of organic solvents in the mobile phase composition and does not require addition of ion pairing agent.

Observed U-shaped retention profiles for basic compounds on fluorinated columns are similar to those observed in the case of silica hydride based stationary phases for polar and nonpolar compounds; this very fact makes it interesting to study the silica hydridebased perfluorinated/fluorinated stationary phase and its retention pattern. It should be noted that all the previously studied fluorinated stationary phases have Type B silica with silanol groups ( $\mathrm{Si}-\mathrm{OH})$ as a backbone. However, in this project, the perfluorinated stationary phase is based on the Type $\mathrm{C}$ silica with a silica hydride backbone ( $\mathrm{Si}-\mathrm{H})$.

\section{H. Surface Modification of Silica}

As mentioned in the earlier section, silica is the preferred backbone for the HPLC stationary phase. The surface of the silica matrix can be modified for HPLC applications. Different techniques of silica modification are well established. Some of the common techniques used for modification of oxides include esterification, chlorination, organosilanization, and reactions using Grignard's reagent. However, some of these 
techniques are not particularly suitable for HPLC applications of silica. For example, reaction products made by esterification can only be used in dry environments or absence of water; hence, it cannot be used for an aqueous normal phase chromatography, which uses water in the mobile phase. In the case of chlorination reaction, the intermediate is hydrolytically unstable and needs to be isolated in dry environments. In addition, the reaction forms salt intermediates, which may interfere in separation applications.

This research project uses the two-step synthesis procedure for the synthesis of modified silica hydride. The first step is silanization which is followed by hydrosilation. This novel approach of silanization/hydrosilation was first introduced by Pesek et al [26]. Silanization and hydrosilation of silica lead to highly stable silica hydride stationary phases where $\mathrm{Si}-\mathrm{H}$ groups replace $95 \%$ of the silanols, thus imparting unique properties to the stationary phase. This method has successfully overcome all the disadvantages of other procedures. Two major steps involved in this method are explained in the following section.

Silanization is the first step of the synthesis, which converts surface silanol ( $\mathrm{Si}-\mathrm{OH})$ groups into silica hydride $(\mathrm{Si}-\mathrm{H})$. A controlled reaction between silica and triethoxysilane (TES) produces weakly hydrophobic silica hydride. This product serves as a stable intermediate for the hydrosilation reaction [21]. Silica hydride has good shelf life and can be stored for a long time without significant decomposition. In this first step, monolayer surface coverage by hydride is achieved. Reaction conditions to be controlled are: concentration of TES, amount of water and acid, and temperature. Figure 6 depicts the schematic of the two-step synthesis procedure. 
In the successive step of hydrosilation, the desired organic moiety is attached on to the surface via $\mathrm{Si}-\mathrm{C}$ bonding. This reaction utilizes an acid catalyst, hexacholoroplatinic acid, also known as Speier's catalyst. It should be noted that the final product of the synthesis has a bonded organic moiety on the surface and the silica hydride groups (SiH). Hence, the separation of solutes on this type of material has the combined influence of the bonded organic moiety as well as the hydrophobic Si-H groups on the surface.

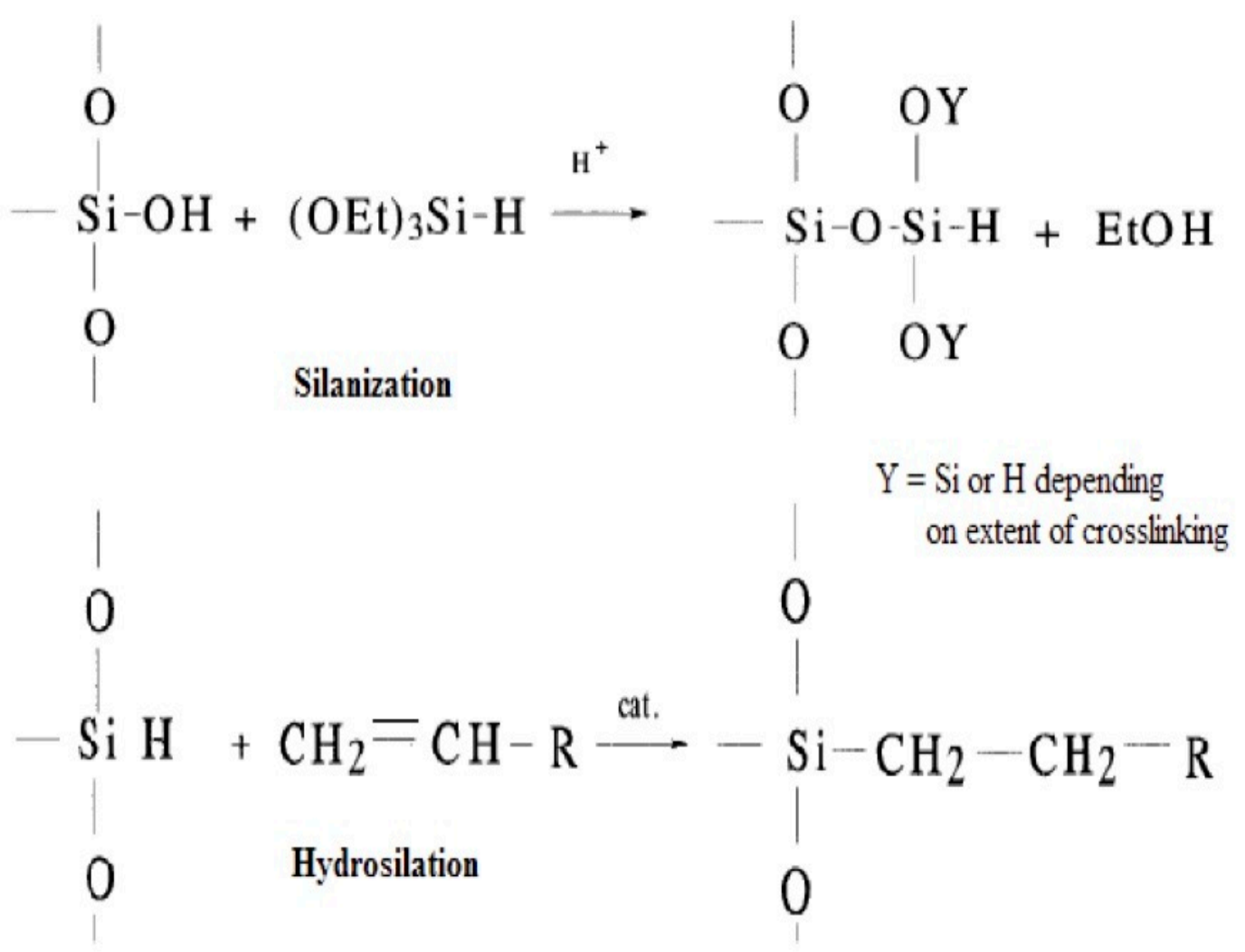
catalyst=hexachloroplatinic acid

Figure 6: Schematic Representation of Silanization/Hydrosilation Procedure. 


\section{Characterization of Modified Silica Hydride}

Various spectroscopic methods and the elemental analysis technique can be used to characterize newly synthesized stationary phase material. In this research, three techniques used for characterization are diffuse reflectance infrared Fourier transform (DRIFT), ${ }^{13} \mathrm{C}$ CP-MAS solid-state NMR, and elemental analysis. This section summarizes basic concepts of the characterization techniques used in this research.

1. Diffuse Reflectance Infrared Fourier Transform (DRIFT)

Diffuse reflectance infrared Fourier transform spectroscopy (DRIFT) is the semiquantitative spectral analysis method mainly used for powdered samples. It confirms the presence of functional groups in the sample. For silica hydride-based samples, the completion of the silanization and hydrosilation, as well as the presence of bonded organic moieties, can be explained qualitatively using DRIFT analysis spectra. Sampling for this technique is easy and convenient.

DRIFT analysis is conducted by focusing infrared light on to the powdered sample; scattered light is collected and relayed to the IR detector [2,3]. A Fourier transform method is used to improve the absorption sensitivity by signal averaging. In this project, DRIFT analysis is performed by irradiating the powdered sample in a small cup. The enhanced signal contains the fingerprint information about the analyte bonded or adsorbed on to the surface.

\section{2. ${ }^{13} \mathrm{C}$ CP-MAS}

NMR is a powerful spectroscopic tool used for structural determination of organic compounds. For the study of surfaces, solid-state NMR is an accepted and frequently 
used method. The solid-state NMR technique can provide detailed information about organic-inorganic materials. It helps to identify their atomic environment and spatial details between various sites.

Generally, in the NMR spectrum of slurry state (solution state) samples, sharp peaks are observed. Sharp peaks are the result of anisotropic cancellation by rapid spinning of nuclei in liquid samples. When the sample is in the solid form, its random movement is restricted and nuclei are somewhat immobilized in one or more directions. Immobilization of nuclei results in a broad peak. Various techniques are employed to reduce peak broadening. Cross-polarization $(\mathrm{CP})$ and magic angle spinning (MAS) are two techniques that enhance the NMR signal for solid samples [22].

In magic angle spinning, a solid sample is subjected to rapid spinning at a particular angle with respect to the external magnetic field. This spinning mimics the natural tumbling of nuclei, resulting in sharp peaks in the spectrum. The specific angle at which sample is spun is referred to as the magic angle, which is generally $55^{0}$.

Poor NMR signals can also be attributed to the low abundance of certain nuclei. In that case, cross-polarization technique is used. In this technique, polarization from abundant spins such as $1 \mathrm{H}$ or $19 \mathrm{~F}$ is transferred to dilute spins such as $13 \mathrm{C}$ or $15 \mathrm{~N}$. The overall effect enhances $\mathrm{S} / \mathrm{N}$. Cross polarization requires that nuclei are dipolar coupled to one another, and it even works while samples are being spun rapidly at the magic angle. In this research, ${ }^{13} \mathrm{C}$ CP-MAS solid-state NMR is used to study the silica hydride-based perfluorinated stationary phase [22]. 


\section{Elemental Analysis}

Elemental analysis is a combustion method mainly used to evaluate the chemical composition of organic compounds. In this method, a sample is subjected to the combustion and combustion fractions are collected in several elemental traps. Carbon elemental analysis can be used for quantitative estimation of silica-based material. The silica matrix of stationary phase material does not contain carbon atoms; hence, the presence of a carbon fraction in the carbon elemental analysis should be attributed to the bonded organic moiety.

Carbon percent values are useful to estimate the surface coverage of a bonded organic moiety. Because there is an increase in the size of bonded phase, there is a decrease in the surface coverage $[27,28]$. The amount of bonded moiety on the silica surface is calculated as the surface coverage $(\alpha)$ in the form of $\mu \mathrm{mol} / \mathrm{m}^{2}$ using the following Berendsen and de Galan equation:

$$
\alpha\left(\mu \mathrm{mol} / \mathrm{m}^{2}\right)=10^{6} p \mathrm{c} /\left(10^{2} \mathrm{M}_{\mathrm{C}} \mathrm{n}_{\mathrm{C}}-p c \mathrm{M}_{\mathrm{R}}\right) \mathrm{S}_{\mathrm{BET}}
$$

In the above equation, $p c-$ carbon $\%$ of the bonded material.

$\mathrm{n}_{\mathrm{C}}$ - number of carbon atoms in bonded organic groups.

$\mathrm{M}_{\mathrm{C}}$ - the atomic weight of carbon.

$\mathrm{M}_{\mathrm{R}}$ - molecular weight of organic compound.

$\mathrm{S}_{\mathrm{BET}}$ - specific surface area of silica material. 


\section{J. Column Evaluation by Variety of Samples}

This section describes various categories of compounds utilized in this project. The ANP and RP dual retention abilities of the silica hydride-based stationary phase were evaluated by using several polar and nonpolar compounds as test probes.

Amino acids are one of the most important classes of biomolecules. They are the building blocks of proteins. Two amino acids, L- tryptophan and L- phenylalanine, were used to probe retention of polar moieties. Amino acids are zwitterions. Based on the $\mathrm{pH}$ of the solution, they can exist in different charged forms. Because of multiple polarities, their separation in RP mode is challenging. Because the ANP mode of chromatography has the ability to separate polar and nonpolar compounds, amino acids are ideal test probes.

The next important classes of polar biomolecules used in this study are nucleobases, nucleotides, and nucleosides. They form the basic units of DNA and RNA. For their separation with the traditional RP mode, strong buffer systems and additives are often used. Strong additives and buffers are incompatible with MS detection. Several nucleobases analyzed on perfluorinated silica hydride based stationary phase were cytosine, adenosine, guanine, thymine, and uracil.

Other miscellaneous polar compounds tested were creatine hydrate, creatinine, and thiamine. Creatine is a nitrogenous organic acid naturally produced in the human body. It is involved in the energy production cycle. Athletes and bodybuilders consume it as a dietary supplement to gain muscle mass. Creatinine is a breakdown product of creatine 
hydrate and is produced in the human body. Separation of these two small polar compounds has been a difficult task.

Thiamine was also used as a small polar test compound. It is vitamin $\mathrm{B}_{1}$, a watersoluble vitamin B-complex and an essential nutrient obtained from the diet. It is a polar compound stable at acidic $\mathrm{pH}$. Thiamine hydrochloride was used for retention studies in the ANP mode.

The last set of compounds used were nonpolar polycyclic aromatic hydrocarbons (PAH). They are aromatic, nonpolar, neutral compounds without further branching. They were used to test RP retention. Toluene was also tested as one of the neutral compounds.

\section{K. Research Goals}

In this research project, the main objective was to synthesize and characterize a silica hydride-based perfluorinated stationary phase and to test its dual retention ability in the reverse-phase as well as normal-phase mode. Synthesis of perfluorinated silica hydride was performed by the hydrosilation process. Previously prepared silica hydride was used as a starting material, and $1 \mathrm{H}, 1 \mathrm{H}, 2 \mathrm{H}$ perfluoro -1 - octene was used as the perfluorinated moiety to be attached on to the surface of silica hydride. Two batches of products were synthesized and reproducibility of the synthetic protocol was confirmed.

Upon completion of each reaction, the newly synthesized stationary phases were subjected to spectroscopic characterization and determination of percent surface coverage. Success of the hydrosilation reaction was confirmed by DRIFT analysis, ${ }^{13} \mathrm{C}$ CP-MAS, and carbon elemental analysis. DRIFT analysis data confirmed the presence of the fingerprint frequencies of the bonded moiety, perfluoro octene. ${ }^{13} \mathrm{C}$ CP-MAS also 
confirmed expected chemical shifts of carbon nuclei. Lastly, percent carbon values obtained from carbon elemental analysis were used to estimate surface coverage by the bonded moiety.

The next step of the project was to evaluate separation behavior of the stationary phase toward hydrophilic and hydrophobic analytes under varied compositions of polar and nonpolar mobile phases. For this purpose, several test compounds with varying polarities were selected to study retention patterns in the ANP and RP modes. Dual retention capability and U-shaped retention profile for polar and nonpolar compounds on silica hydride based stationary phase was established. Figure 7 shows an ideal retention profile plot for silica hydride-based stationary phases, when using a mixture of polar and nonpolar solvents as the mobile phase.

The next important objective of this project was to study the effect of varying $\mathrm{pH}$ on the retention time of compounds. Two different buffer systems, formic acid and ammonium acetate, were used in this study. The relationship was established between the retention time of solutes and $\mathrm{pH}$ of the mobile phase at different buffer concentrations.

Lastly, compatibility of acetone as an organic solvent in the mobile phase was tested. Considering some of the advantages of acetone over commonly used acetonitrile, it is useful to study acetone as an organic solvent in the mobile phase. 


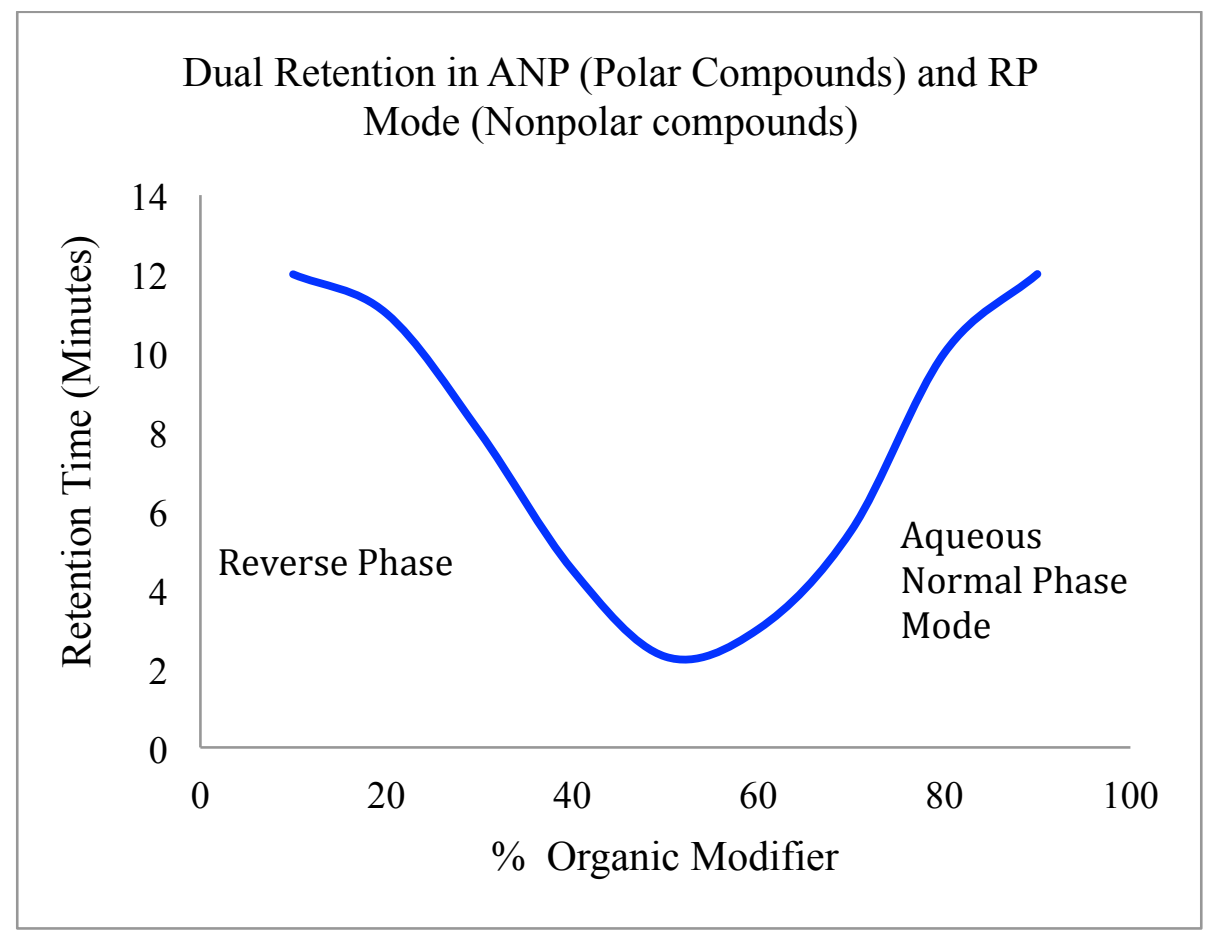

Figure 7: Generic Plot for Retention Profile of Silica Hydride-based Stationary Phase. 


\section{EXPERIMENTAL}

\section{A. Materials}

The chemicals used in the preparation of stationary and mobile phases utilized in this research are tabulated in this section. The small polar molecules and nonpolar compounds used in the evaluation of columns are also listed in the following tables.

\section{Table 1: Material used for Hydrosilation.}

\begin{tabular}{|c|c|}
\hline Chemical Name & Manufacturing Company \\
\hline 1H, 1H, 2H Perfluoro-1-octene & Aldrich Chemical Co. \\
\hline Hexachloroplatinic Acid & Sigma-Aldrich \\
\hline Toluene & Fisher Chemicals \\
\hline Diethyl ether & Sigma-Aldrich \\
\hline Dichloromethane & Fisher Chemicals \\
\hline
\end{tabular}


Table 2: Chemicals Required for Mobile Phase Preparation.

\begin{tabular}{|c|c|}
\hline Chemical Name & Manufacturing Company \\
\hline Acetonitrile & Honeywell \\
\hline Acetone (HPLC grade) & Burdick and Jackson \\
\hline Formic Acid & Spectrum mfg. corp. \\
\hline Ammonium Acetate & Matheson Coleman \& Bell \\
\hline
\end{tabular}

Table 3: Samples Analyzed for ANP Retention of Stationary Phase.

Table 3.1: Nucleobases, Nucleosides, and Nucleotides.

\begin{tabular}{|c|c|}
\hline Chemical Name & Manufacturing Company \\
\hline Cytosine & Nutritional Biochemical Corp. \\
\hline Adenosine & Calbiochem Co. \\
\hline Uracil & Sigma Chemical Co. \\
\hline Thymine & Nutritional Biochemical Corp. \\
\hline Guanine & NBC National Biochemical Corp. \\
\hline
\end{tabular}

Table 3.2: Amino Acids.

\begin{tabular}{|c|c|}
\hline Chemical Name & Manufacturing Company \\
\hline L-Phenylalanine & J.T. \& Baker Chemical Co. \\
\hline L-Tryptophan & Sigma Chemical Co. \\
\hline
\end{tabular}


3.3 Miscellaneous Compounds.

\begin{tabular}{|c|c|}
\hline Chemical Name & Manufacturing Company \\
\hline Creatinine & Sigma Chemical Co. \\
\hline Creatine Hydrate & Sigma Chemical Co. \\
\hline Thiamine Hydrochloride & Sigma Chemical Co. \\
\hline
\end{tabular}

Table 4: Samples Analyzed for RP Retention of Stationary Phase.

Polycyclic Aromatic Hydrocarbons (PAH), Nonpolar Compounds.

\begin{tabular}{|c|c|}
\hline Chemical Name & Manufacturing Company \\
\hline Pyrene & Aldrich \\
\hline Fluorene & Aldrich \\
\hline Phenanthrene & Aldrich \\
\hline Naphthalene & J.T. \& Baker Chemical Co. \\
\hline Toluene & Fisher Chemicals \\
\hline
\end{tabular}




\section{B. Structures}

\section{Perfluorinated Moiety Bonded to Silica Hydride (Hydrosilation)}

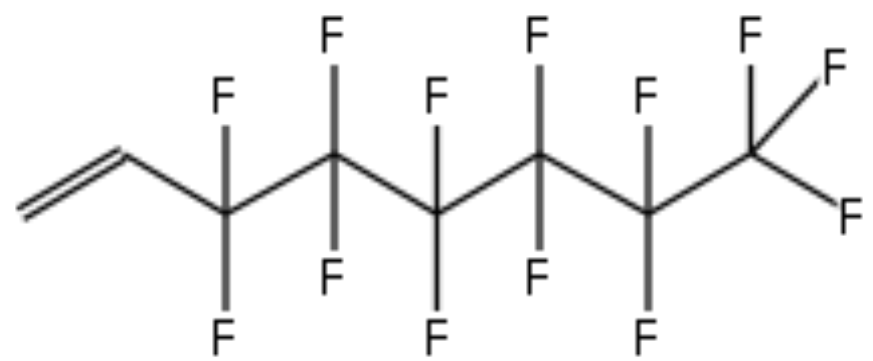

$1 \mathrm{H}, 1 \mathrm{H}, 2 \mathrm{H}$ - Perfluoro - 1 - Octene

\section{Samples Analyzed for ANP Retention}

2.1. Nucleobases, Nucleotides, and Nucleosides<smiles>Nc1cc[nH]c(=O)n1</smiles>

Cytosine<smiles>O=c1cc[nH]c(=O)[nH]1</smiles>

Uracil

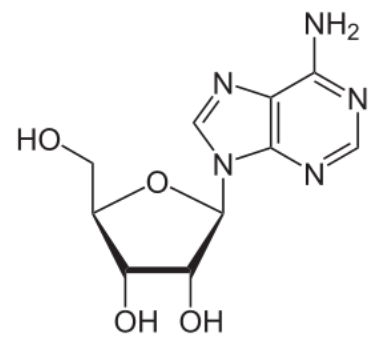

Adenosine<smiles>Cc1c[nH]c(=O)[nH]c1=O</smiles>

Thymine<smiles>Cc1c[nH]c(=O)[nH]c1=O</smiles>

Thymine 
<smiles>N[C@@H](Cc1ccccc1)C(=O)O</smiles>

L-Phenylalanine

2.3. Miscellaneous compounds

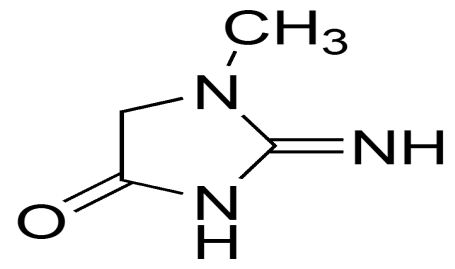

Creatinine<smiles>Cc1ncc(C[n+]2csc(CCO)c2C)c(N)n1</smiles>

Thiamine Hydrochloride<smiles>N[C@@H](Cc1c[nH]c2ccccc12)C(=O)O</smiles>

L-Tryptophan

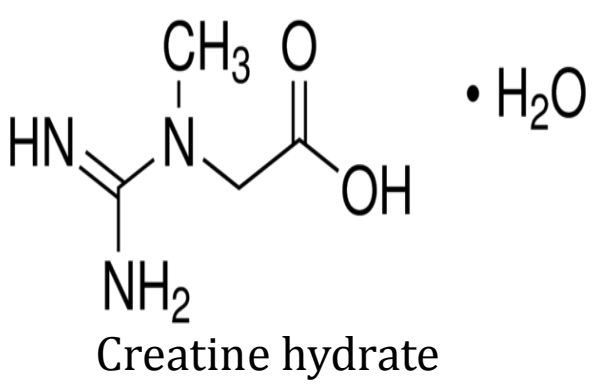


3. Samples Analyzed for RP Retention<smiles>[R]c1ccc2ccc3cccc4ccc1c2c34</smiles>

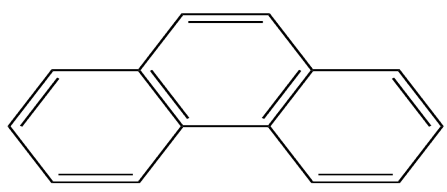

Phenanthrene

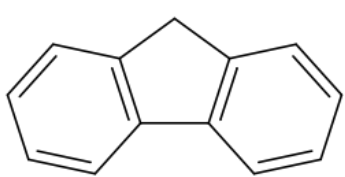

Fluorene<smiles>c1ccc2ccccc2c1</smiles>

Naphthalene 


\section{Synthetic Procedure}

The synthesis of perfluorinated silica hydride is a two-step process. Silanization is the first step, which involves conversion of silanol groups into the silica hydride. Previously synthesized silica hydride was used in this project. The second step is known as hydrosilation, which was used to bond the perfluorinated organic moiety on to the surface of silica hydride. The detailed procedure for the hydrosilation reaction is described in the following section.

1. Hydrosilation

All of the required glassware was kept in the oven for drying overnight. Glassware used for the synthesis mainly included a $500 \mathrm{~mL}$ round bottom flask, a condenser, a magnetic stirrer, and a glass stopper. Accurately weighed 5 grams of silica hydride was dried overnight at $110^{\circ} \mathrm{C}$ in a vacuum oven.

A $500 \mathrm{~mL}$ three-necked round-bottom (RB) flask was attached to the condenser. The condenser had a drying tube, a thermometer, and a dried glass stopper attached to it. Water condenser tubes were properly attached to a running water source. The roundbottom flask was kept into the heating mantle, which was placed on to the stirring plate. $10 \mathrm{~mL}$ of $1 \mathrm{H}, 1 \mathrm{H}, 2 \mathrm{H}$ - perfluoro - 1 - octene was dissolved in $200 \mathrm{~mL}$ of toluene and then it was carefully transferred to the round-bottom flask. A magnetic stirrer of suitable size was placed in the round-bottom flask. Subsequently, $1 \mathrm{~mL}$ of $10 \mathrm{mM}$ hexachloroplatinic acid in isopropanol was added to the RB flask. The reaction mixture was then heated to $70^{\circ} \mathrm{C}$ for one hour with constant stirring. 
After one hour, 5 grams of vacuum dried silica hydride was carefully and slowly added to the RB flask with constant stirring. The temperature was then raised to $100^{\circ} \mathrm{C}$ and it was maintained for 100 hours. Maintaining this reaction mixture at $100^{\circ} \mathrm{C}$ for 100 hours was essential for the temperature to be stable. For this purpose, reaction conditions were monitored after every 20 to 24 hours.

Once the reaction mixture was cooled down completely, the product was filtered using vacuum suction. The filtered product was washed successively with $50 \mathrm{~mL}$ of toluene, 50 $\mathrm{mL}$ dichloromethane, and $50 \mathrm{~mL}$ of diethyl ether. The washed product was then dried at room temperature overnight and later it was vacuum dried at $100^{\circ} \mathrm{C}$ for 24 hours.

To confirm the reproducibility of the synthesis protocol and to meet the requirement of more product for further analysis, the second batch of product was synthesized. A similar procedure was followed for the synthesis of the second batch except that quantities of starting materials were altered; $3 \mathrm{~mL}$ of $1 \mathrm{H}, 1 \mathrm{H}, 2 \mathrm{H}$ - perfluoro - 1 - octene was dissolved in $60 \mathrm{~mL}$ toluene and transferred it to the $250 \mathrm{~mL}$ of RB flask. Subsequently, $300 \mu \mathrm{L}$ of $10 \mathrm{mM}$ hexachloroplatinic acid in isopropanol was added to the RB flask. After heating this mixture at $70^{\circ} \mathrm{C}$ for 1 hour, 1.5 grams of vacuum dried silica hydride was added to the reaction mixture with constant stirring. This reaction mixture was refluxed for 100 hours at $100^{\circ} \mathrm{C}$. The reaction product was washed with a series of solvents, as explained earlier. The washed product was then dried at room temperature overnight and later vacuum dried at $100^{\circ} \mathrm{C}$ for 24 hours. Figure 8 shows the actual experimental setup for hydrosilation reaction. 


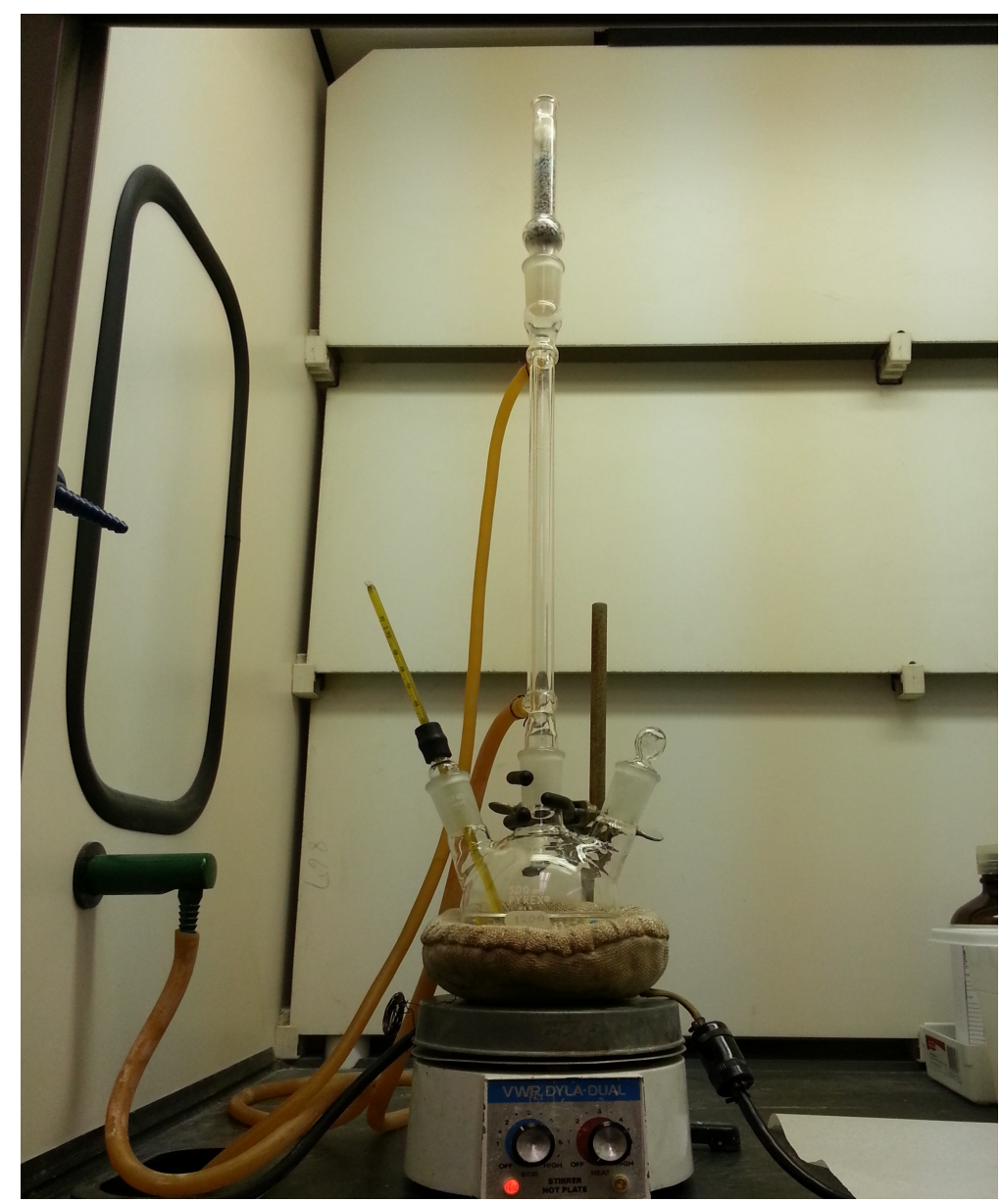

Figure 8: Experimental Setup for Hydrosilation Reaction. 


\section{Column Packing}

A synthesis product of batch one was packed into the column by MicroSolv, New Jersey. Column details are as stated below;

Chain length - Pefluoro C 8

Pore size $\quad-100 \AA$

Particle size $-4 \mu \mathrm{m}$

Dimension $-4.6 \times 75 \mathrm{~mm}$

$\mathrm{pH}$ range $\quad-2.5$ to 7

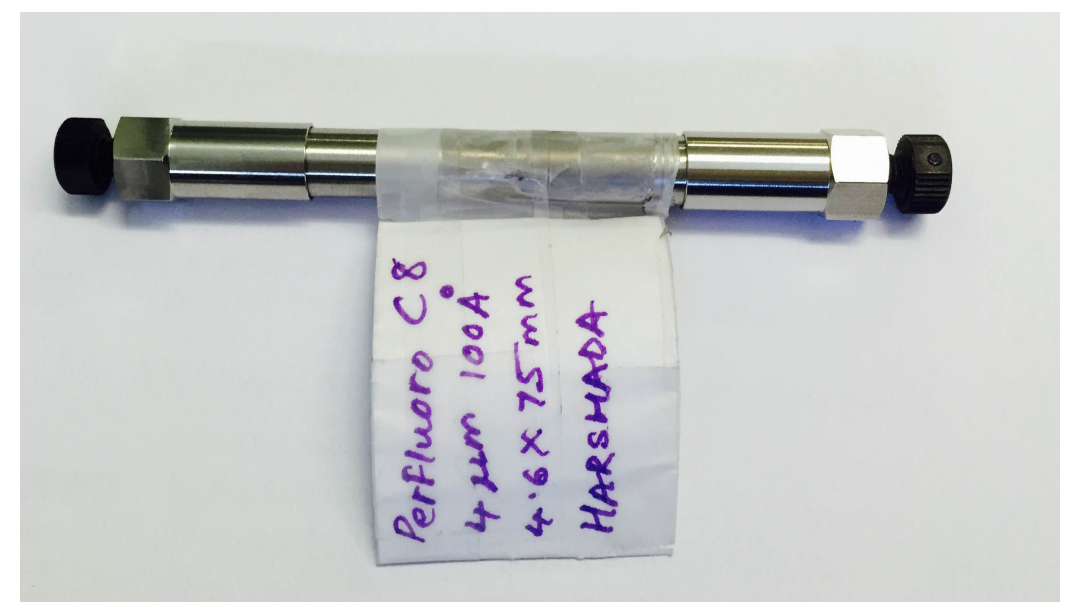

Figure 9: Perfluoro C8 Column. 


\section{Instrumental Procedures}

1. Diffuse Reflectance Infrared Fourier Transform (DRIFT) Analysis

DRIFT spectra of batch 1 and batch 2 synthesis products were obtained using an ATI

Mattson Infinity series FTIR ${ }^{\mathrm{TM}}$ instrument. This instrument is equipped with a deuterated triglycine sulfate (DTGC) detector. The sample was placed in a sample cup, and the upper surface of the sample was smoothed by a spatula. A background signal was first collected, and then the sample cup was carefully placed in the instrument. The spectrum was recorded for both batches separately. A scan for percent transmittance $(\% \mathrm{~T})$ was obtained over the range of 500 to $4000 \mathrm{~cm}^{-1}$.

\section{2. ${ }^{13} \mathrm{C}$ CP-MAS}

The ${ }^{13} \mathrm{C}$ CP MAS solid-state NMR spectrum of the perfluorinated product was obtained from our collaborator at the Chemistry Department, San Francisco State University (San Francisco, CA). A Bruker DRX 300 MHz instrument was used for NMR studies. The spectrum utilized the combined techniques of cross-polarization and magic angle spinning.

\section{Carbon Elemental Analysis}

The carbon elemental analysis by combustion provided percentages of carbon present in the given sample. Small amounts of synthetic product of batch 1 and batch 2 were sent to the ALS Environmental Laboratory and Galbraith Laboratories, respectively. The Berendsen and De Galan equation was used to calculate surface coverage of perfluorinated bonded moiety on to the silica hydride. 


\section{High Performance Liquid Chromatography (HPLC)}

A Hewlett Packard/Agilent 1050 series HPLC instrument was used for this study. The system was equipped with an Alltech on-line degasser, VW UV detector, auto sampler, and a quaternary pump. The ChemStation software was used for the purpose of instrument control and data collection. An HP deskjet printer was used to print the chromatograms. The deionized water used for mobile phases was prepared on a MilliQTM purification system (Millipore Corp., Bedford, MA). For a majority of the samples analyzed by HPLC-UV, the mobile phase flow rate was $0.5 \mathrm{~mL} / \mathrm{min}$. Figure 10 shows the HPLC instrument used for analysis.

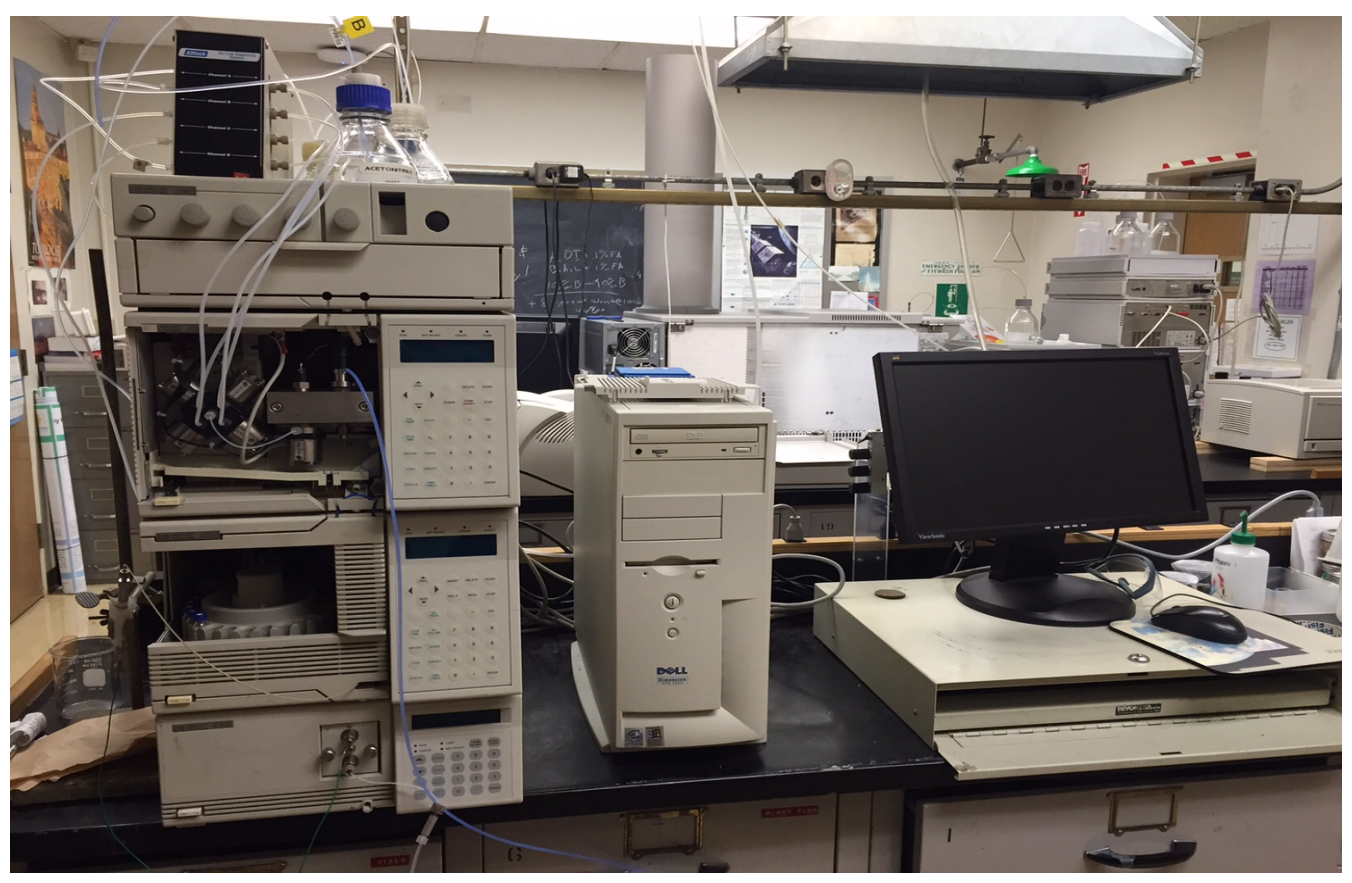

Figure 10: HP/Agilent 1050 Series HPLC-UV Instrument. 
An Agilent TOF LC/MS instrument was used for mass spectrometric analysis. ChemStation software was used for desired mass calculation and data analysis. MS detection was mainly used for acetone-based studies. LC/MS grade solvents were used. For all the samples analyzed by LC/MS, the mobile phase flow rate was set at 0.7 $\mathrm{mL} / \mathrm{min}$. Figure 11 shows the Agilent 1100 series (TOF) LC-MS instrument used for analysis.

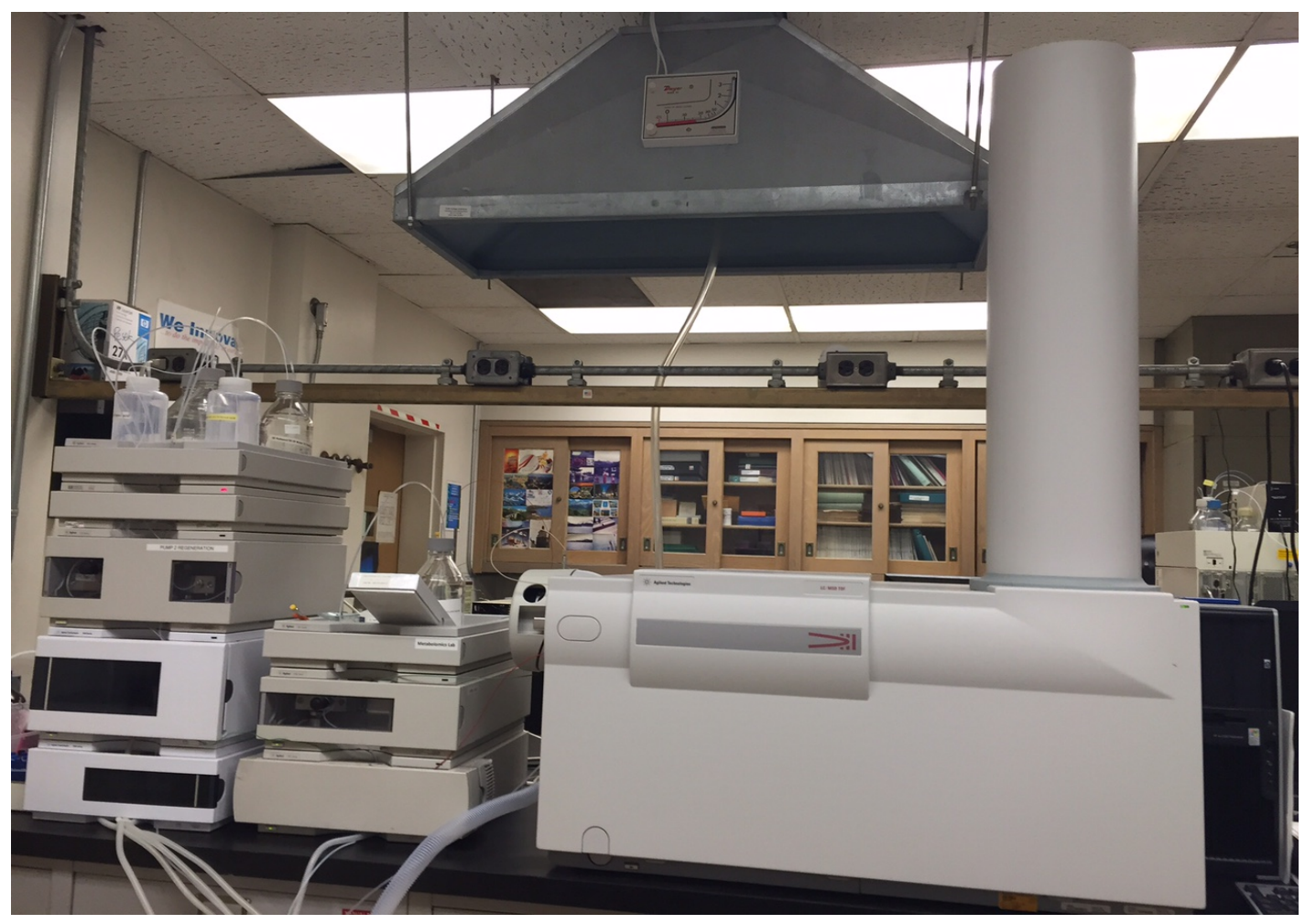

Figure 11: Agilent 1100 series (TOF) LC-MS. 


\section{RESULTS AND DISCUSSION}

\section{A. DRIFT Spectroscopic Analysis}

Completion of the hydrosilation reaction was confirmed by DRIFT spectroscopic analysis. Figure 12 shows the DRIFT spectrum of the batch 1 synthetic product (perfluorinated silica hydride). A strong peak at $2250 \mathrm{~cm}^{-1}$ is due to the stretching vibration of a silica hydride bond $(\mathrm{Si}-\mathrm{H})$. The presence of intense peak at $2250 \mathrm{~cm}^{-1}$ confirms the presence of $\mathrm{Si}-\mathrm{H}$ bonds on the surface.

A small and sharp peak at $2950 \mathrm{~cm}^{-1}$ is due to the vibrational frequency of aliphatic C$\mathrm{H}$ bonds. The appearance of this frequency in the spectrum confirms the presence of fluorinated organic moiety on the surface. It also confirms that bonding between silica hydride and fluorinated organic moiety is successful. The carbon-fluorine bond (C-F) has a characteristic stretching frequency in the range of $950 \mathrm{~cm}^{-1}$ to $1150 \mathrm{~cm}^{-1}$. However, this region is dominated by silanol $(\mathrm{Si}-\mathrm{OH})$ bond frequencies. Therefore, the signal for $\mathrm{C}-\mathrm{F}$ bond cannot be directly observed. Si-OH dominates the matrix of silica material, and hence they have a strong presence in the spectrum.

Figure 13 shows the spectrum of the batch 2 synthetic product. Similar characteristic frequencies of $\mathrm{Si}-\mathrm{H}$ at $2250 \mathrm{~cm}^{-1}$ and $\mathrm{C}-\mathrm{H}$ at $2950 \mathrm{~cm}^{-1}$ are present in this spectrum as well. Figure 14 shows the overlap of batch 1 and batch 2 spectra. DRIFT spectra of batch 1 and 2 products are quite comparable, which proves that the synthesis protocol is reproducible with a similar bonding pattern. 


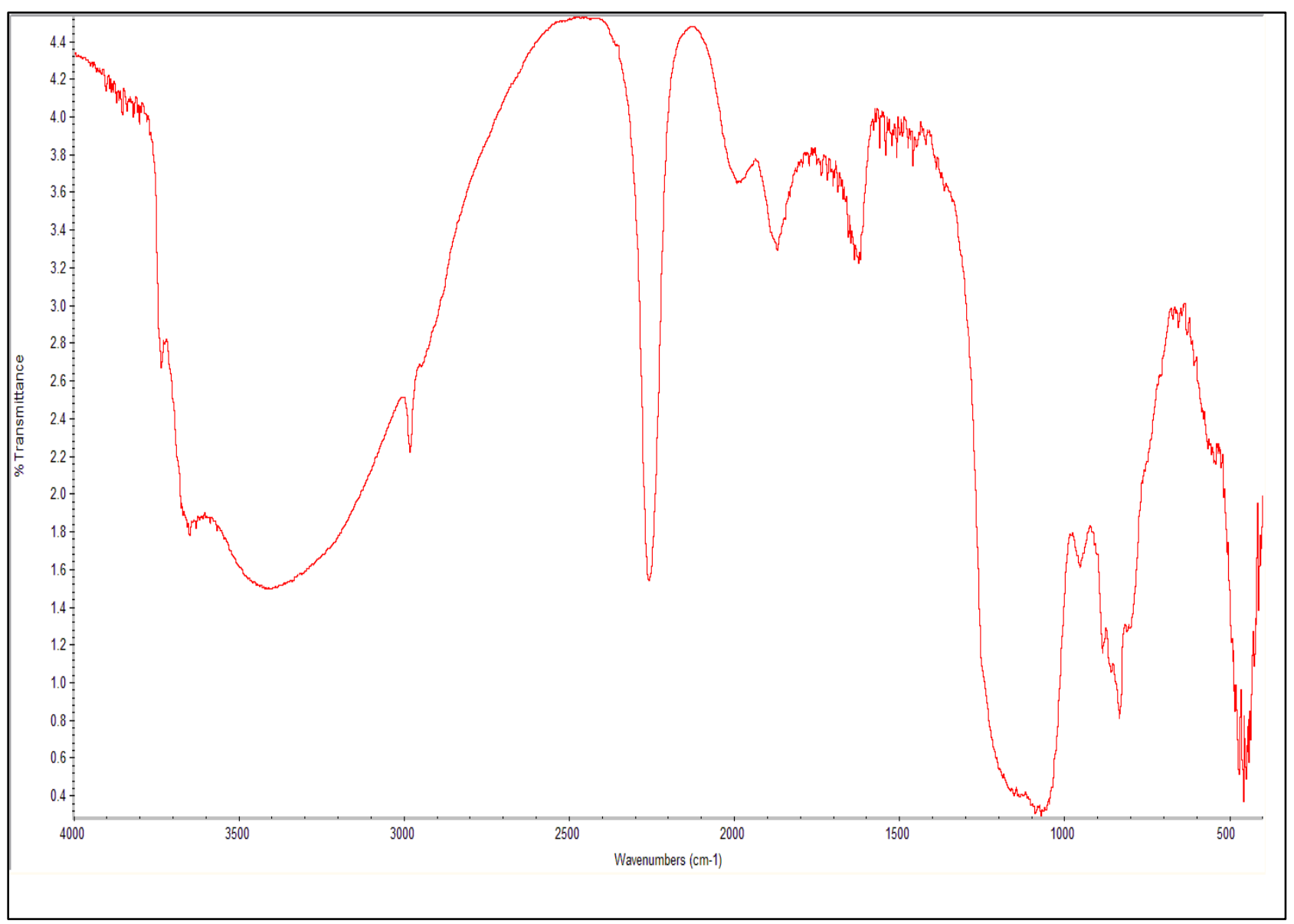

Figure 12: DRIFT Spectrum of Perfluorinated Silica Hydride Batch 1. 


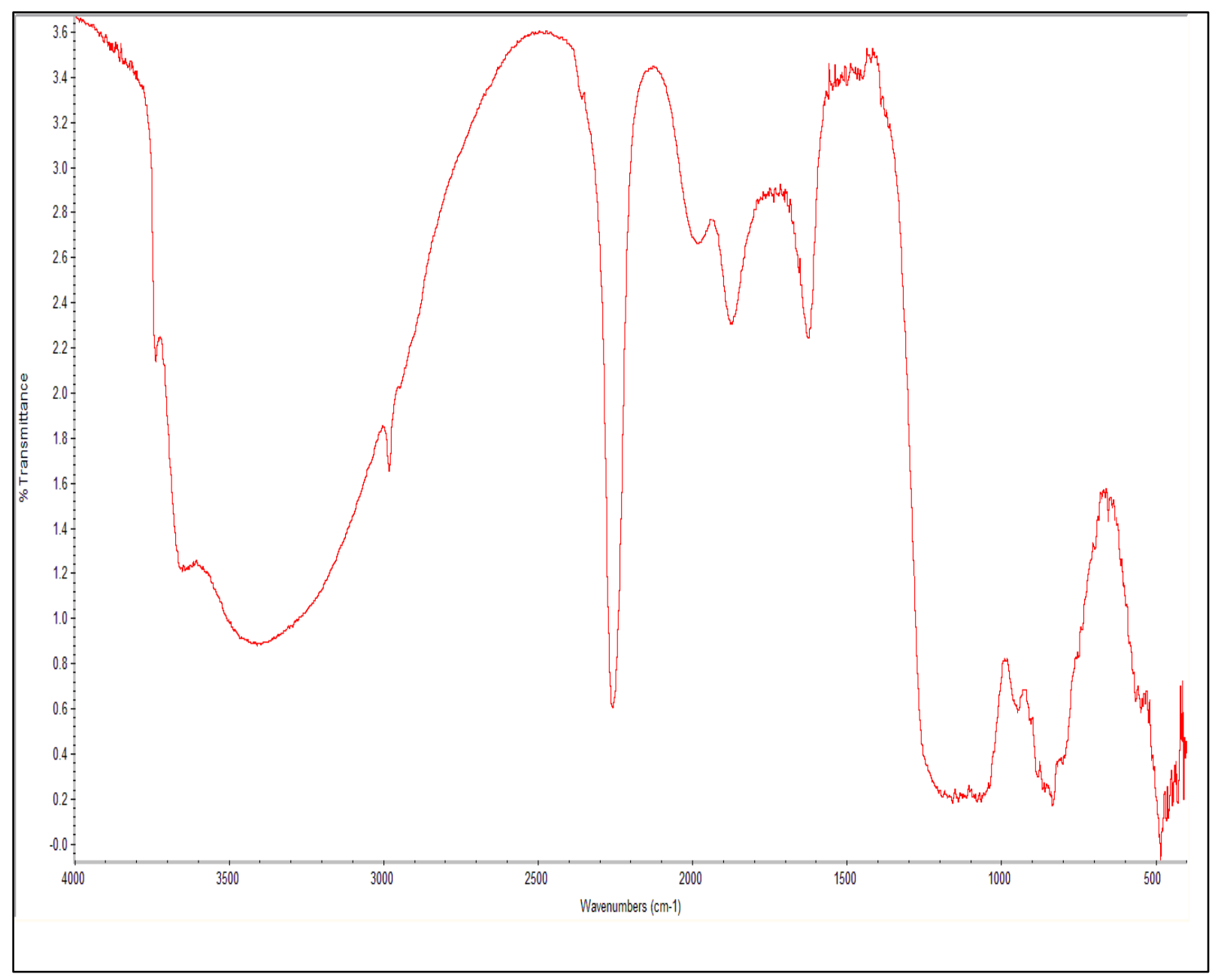

Figure 13: DRIFT Spectrum of Perfluorinated Silica Hydride Batch 2. 


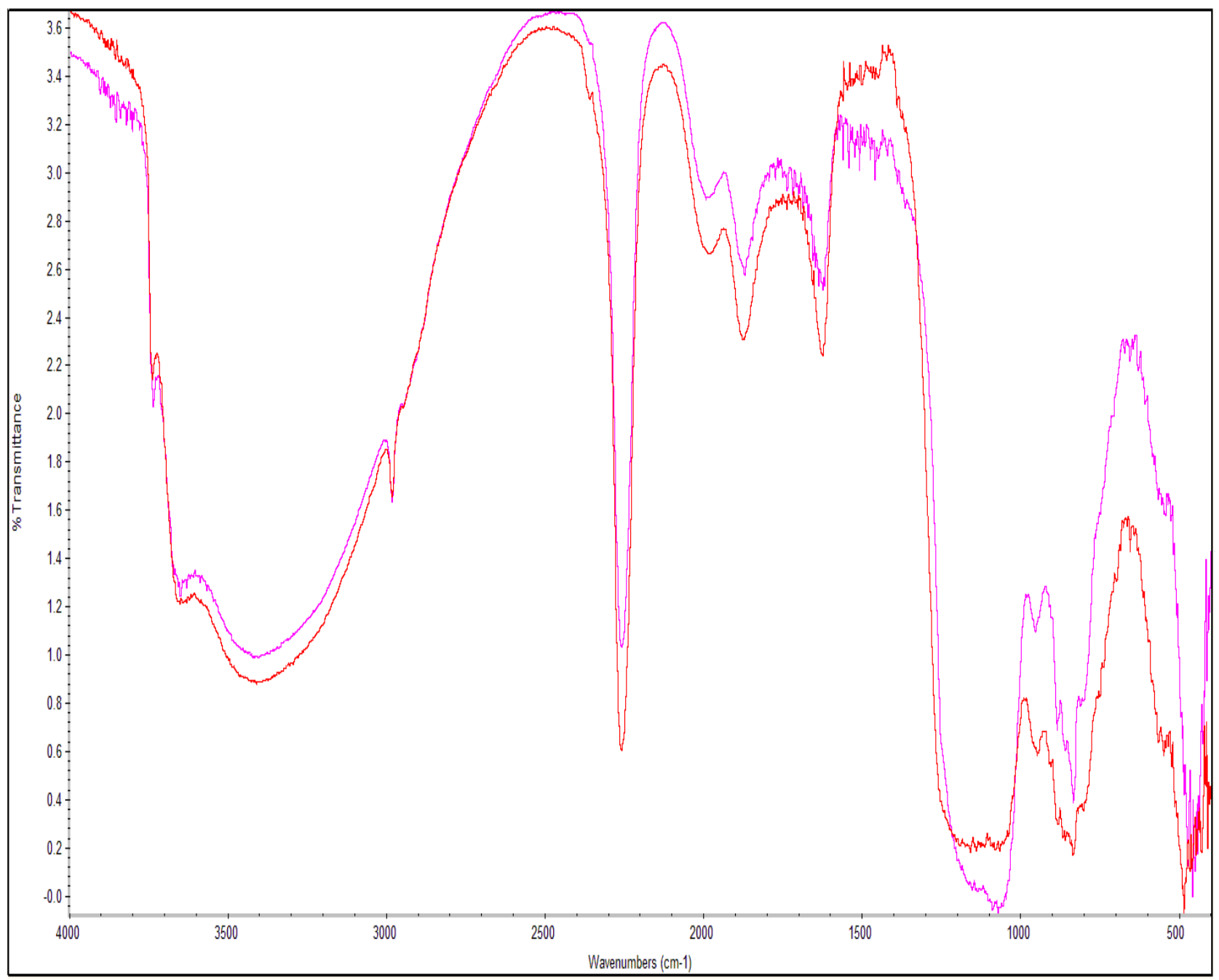

: Batch $1=$ : Batch 2

Figure 14: Overlap of DRIFT Spectra of Perfluorinated Silica Hydride Batch 1 and

Batch 2. 


\section{B. Carbon Elemental Analysis}

The presence of an organic moiety was confirmed by carbon elemental analysis. This technique was mainly used to have a quantitative estimate of percentage of carbon present on the surface of the stationary phase. The surface coverage of carbon was calculated by the Berendsen and De Galan equation. Information about the surface coverage and carbon load (\% carbon) is important to understand the retention behavior and possible separation mechanism for any particular stationary phase.

Low surface coverage was achieved for both batches of synthesis products, which is favorable to examine the combined influence of silica hydride and perfluorinated moiety with respect to the retention of solutes with varying polarities. Table 5 summarizes carbon analysis values including percent coverage obtained from ALS Environmental and Galbraith laboratories and surface coverage of carbon in $\mu \mathrm{mol} / \mathrm{m}^{2}$.

Table 5: Surface Coverage of Bonded Organic Moiety.

\begin{tabular}{|l|l|l|c|c|c|c|}
\hline & Silica & $\begin{array}{c}\mathrm{S}_{\mathrm{BET}} \\
\left(\mathrm{m}^{2} / \mathrm{g}\right)\end{array}$ & Bonding Moiety & Molecular Formula & $\% \mathrm{C}$ & $\begin{array}{c}\text { Surface } \\
\text { Coverage } \\
\mu \mathrm{mol} / \mathrm{m}^{2}\end{array}$ \\
\hline Batch 1 & Atrosil & 350 & perfluoro-1-octene & $\mathrm{CF}_{3}\left(\mathrm{CF}_{2}\right)_{5} \mathrm{CH}=\mathrm{CH}_{2}$ & 1.55 & 0.488 \\
\hline Batch 2 & Atrosil & 350 & perfluoro-1-octene & $\mathrm{CF}_{3}\left(\mathrm{CF}_{2}\right)_{5} \mathrm{CH}=\mathrm{CH}_{2}$ & 2.20 & 0.710 \\
\hline
\end{tabular}




\section{Solid-State NMR $-{ }^{13} \mathrm{C}$ CP-MAS}

Solid-state NMR is the most promising tool to confirm structure and bonding of solid material. Figure 15 shows the ${ }^{13} \mathrm{C}$ CP-MAS spectrum of silica hydride bonded with $1 \mathrm{H}$, $1 \mathrm{H}, 2 \mathrm{H}$ - perfluoro -1 - octene. There are two important peaks in this spectrum that are characteristic of the sample. Although the peaks are not as sharp as solution spectra, they are sufficient to identify specific chemical shifts.

The first characteristic peak is at $2 \mathrm{ppm}$, which represents a methylene carbon directly attached to the silicon atom (carbon \# 1 in Figure 15). The second characteristic peak appears at $24 \mathrm{ppm}$, which represents the second methylene carbon in the chain (carbon \#2 in Figure 15). The appearance of these two characteristic peaks in the spectrum further strengthens the conclusion that there is a successful bonding between silica hydride and perfluorinated moiety. Peak 1 has been observed in previously synthesized silica hydridebased materials.

Because the ${ }^{13} \mathrm{C} C \mathrm{CP}-\mathrm{MAS}$ technique involves proton decoupling, only those carbon atoms attached to hydrogen ( $\mathrm{C} \# 1$ and $\mathrm{C} \# 2$ in Figure 16) show up in the spectrum. Carbon atoms attached to the fluorine cannot be observed in the CP-MAS spectrum.

Peaks that appear between 60 to $80 \mathrm{ppm}$ are the result of residual solvents. The solvents used for washing of synthesis product may have some traces left, which also appear in the spectrum. 


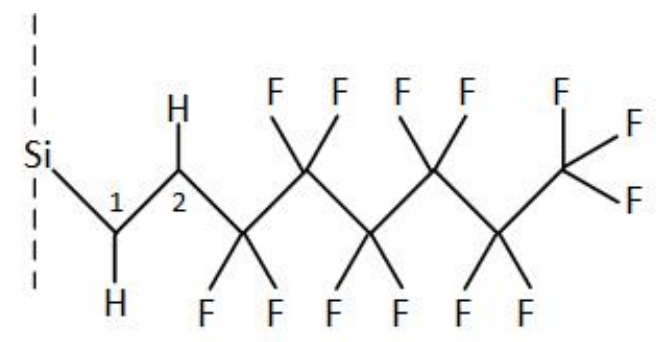

Figure 15: Bonding Between Si and Perfluorinated Moiety.

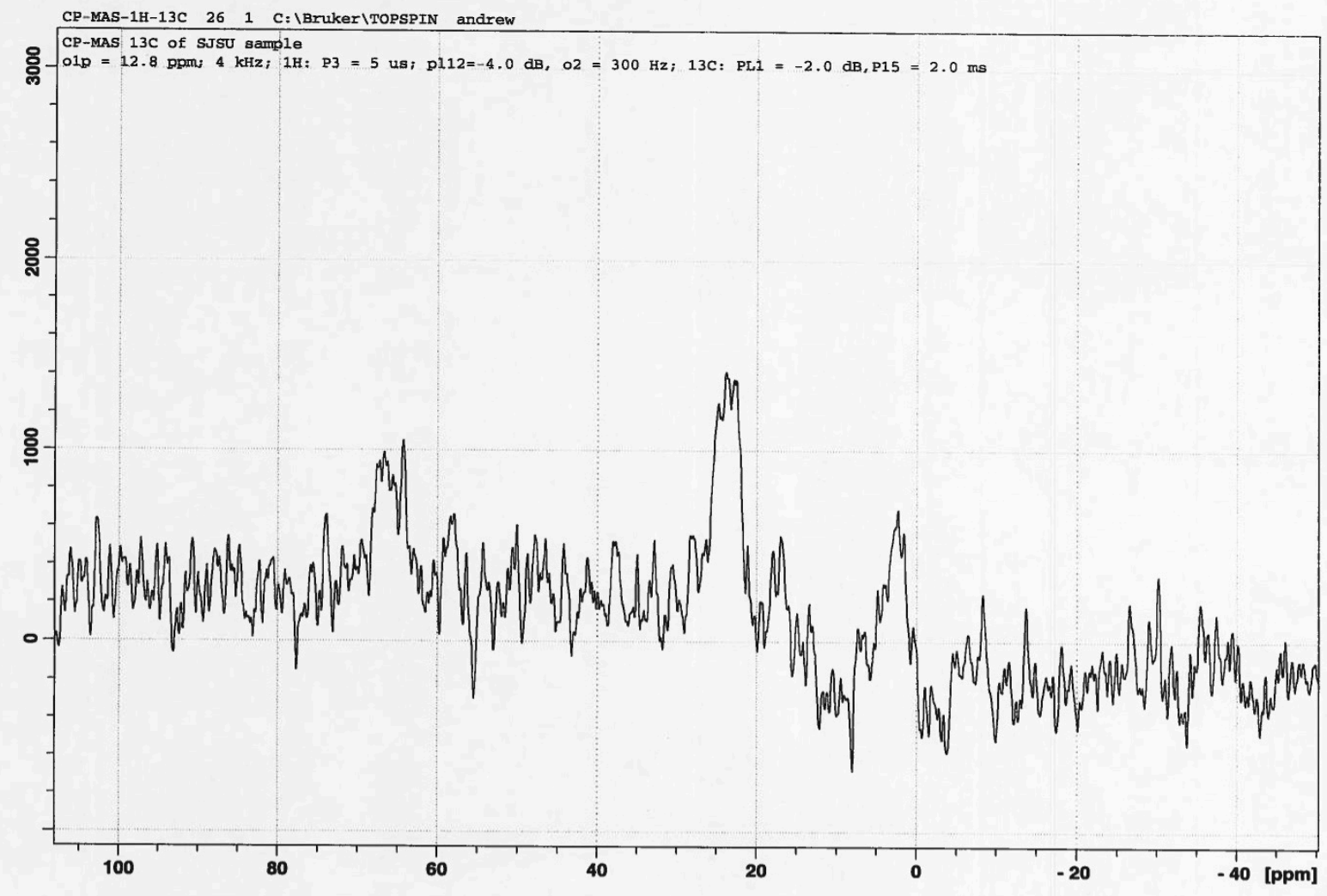

Figure 16: ${ }^{13} \mathrm{C}$ CP-MAS Spectrum of Perfluoro Silica Hydride. 


\section{Chromatographic Characterization of Stationary Phase}

The primary goal of this project was to identify chromatographic separation patterns for the silica hydride-based perfluorinated stationary phase and to test a dual (ANP and RP) retention capability of the stationary phase. Several test samples with varying polarities were analyzed with respect to their retention times. The most important objective was to test ANP retention for small polar solutes. The retention profile of different samples was studied using UV-Vis and MS detectors. The following section discusses retention profiles of different classes of compounds.

\section{ANP Retention Profile}

Silica hydride-based material has shown good retention for polar compounds in the past [12]; hence, it was one of the goals to check the trend for retention of polar solutes on the perfluorinated silica hydride-based stationary phase. Different classes of compounds tested for ANP retention were amino acids, nucleobases, ergogenic acids, and certain miscellaneous compounds.

An HPLC column packed with the newly synthesized stationary phase was first conditioned by running 50:50 acetonitrile: DI water for half an hour. In this study, every injection was done twice or sometimes three times; hence, the final reading for retention time is the average of multiple injections. Sample preparation procedures and other chromatographic conditions are discussed in this section.

\subsection{Amino Acids}

Amino acids are biologically important hydrophilic compounds. They serve as building units of proteins. In this project, two amino acids analyzed were: L-tryptophan 
and L-phenylalanine. The mobile phase for each run was composed of a mixture of polar and nonpolar solvents. Generally, in HPLC method development, polar solvent is referred to as solvent A and nonpolar solvent is referred to as solvent $\mathrm{B}$. In this research, the polar solvent used was DI water (solvent A) and the nonpolar solvent was acetonitrile (solvent B). Five hundred $\mathrm{mL}$ of each solvent $\mathrm{A}$ and $\mathrm{B}$ were prepared separately, and $0.1 \% \mathrm{v} / \mathrm{v}$ formic acid was added to both the solvents as buffer. Sample preparation for amino acids L-tryptophan and L-phenylalanine was not crucial. Both these compounds were readily dissolved in a 50:50 A:B solvent composition. Samples were prepared by dissolving $1 \mathrm{mg}$ of the compound in $1 \mathrm{~mL}$ of 50:50 A:B solvent composition along with $0.1 \% \mathrm{v} / \mathrm{v}$ formic acid. Chromatographic analysis involved retention time measurements at varying composition of solvents A and B, starting with A:B 90:10 through A:B 20:80 in 10 percent increments. The flow rate for each run was optimized to $0.5 \mathrm{~mL} /$ minute. The injection volume was optimized by multiple injections and evaluating their respective peak heights. The optimized injection volume was set to $4 \mu \mathrm{L}$. The UV detector was used for this study with $254 \mathrm{~nm}$ as the wavelength of detection. Table 6 summarizes the retention time in minutes at different compositions of mobile phase. 
Table 6: Retention Time for Amino Acids.

\begin{tabular}{|c|c|c|c|}
\hline \multicolumn{2}{|c|}{ Concentration (\%) } & \multicolumn{2}{c|}{$\begin{array}{c}\text { Retention Time } \\
\text { (Minutes) }\end{array}$} \\
\hline $\begin{array}{c}\text { DI Water }+ \\
0.1 \% \text { Formic Acid }\end{array}$ & $\begin{array}{c}\text { Acetonitrile }+ \\
0.1 \% \text { Formic Acid }\end{array}$ & L- Phenylalanine & L-Tryptophan \\
\hline 70 & 30 & 2.622 & 3.00 \\
\hline 60 & 40 & 2.625 & 3.089 \\
\hline 50 & 50 & 2.692 & 3.353 \\
\hline 40 & 60 & 3.282 & 3.72 \\
\hline 30 & 70 & 4.639 & 3.96 \\
\hline 20 & 80 & 8.699 & 9.541 \\
\hline 15 & 85 & 11.921 & 15.067 \\
\hline
\end{tabular}

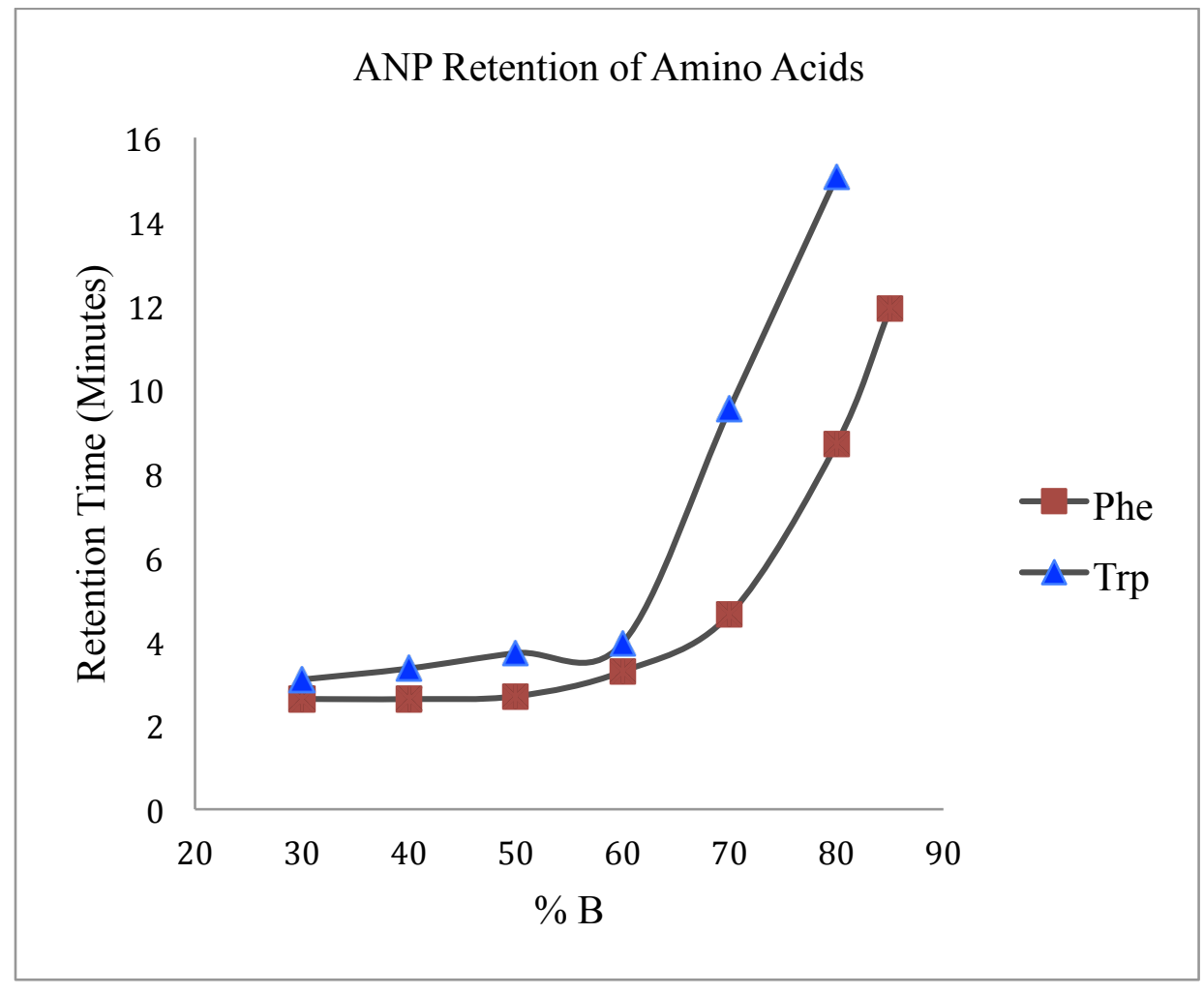

Figure 17: ANP Retention of Amino Acids. 
Figure 17 shows the graph of retention times as a function of percent of solvent $\mathrm{B}$ for Lphenylamine and L-tryptophan. Retention times in minutes are plotted on the Y-axis verses $\% \mathrm{~B}$ on the $\mathrm{X}$-axis. From Figure 17, it is evident that both these amino acids exhibit an ANP retention pattern. Polar amino acids are very well retained on a silica hydridebased perfluorinated stationary phase. With an increase in the percent of nonpolar solvent in the mobile phase, there is an increase in the retention time for L-phenylalanine and Ltryptophan.

\subsection{Nucleobase related compounds}

Nucleobases, nucleotides, and nucleosides are important classes of biomolecules. They function as building blocks of DNA and RNA. Test compounds used under this category were adenosine, thymidine, cytosine, guanine, and uracil. In this study, solvent A was DI water and solvent B was acetonitrile with $0.1 \%$ formic acid as buffer. All the samples were prepared by dissolving $1 \mathrm{mg} / \mathrm{mL}$ of $50: 50 \mathrm{~A}: \mathrm{B}$ except for guanine. It was dissolved in a $200 \mu \mathrm{L} \mathrm{HCl}$ solution containing 50:50 DI water: acetonitrile premixed with $0.1 \%$ formic acid to make a $1 \mathrm{mg} / \mathrm{mL}$ sample solution. Each sample vial was sonicated for 1 minute to ensure complete dissolution of the sample. The optimized injection volume was $5 \mu \mathrm{L}$. The flow rate was $0.5 \mathrm{~mL} /$ minute. The $\mathrm{UV}$ detector was used and the selected wavelength of detection was $254 \mathrm{~nm}$. Tables 7 and 8 list different compositions

of the mobile phases and their respective retention times in minutes for five different test samples. 
Table 7: Retention Time for Adenosine, Cytosine, and Guanine.

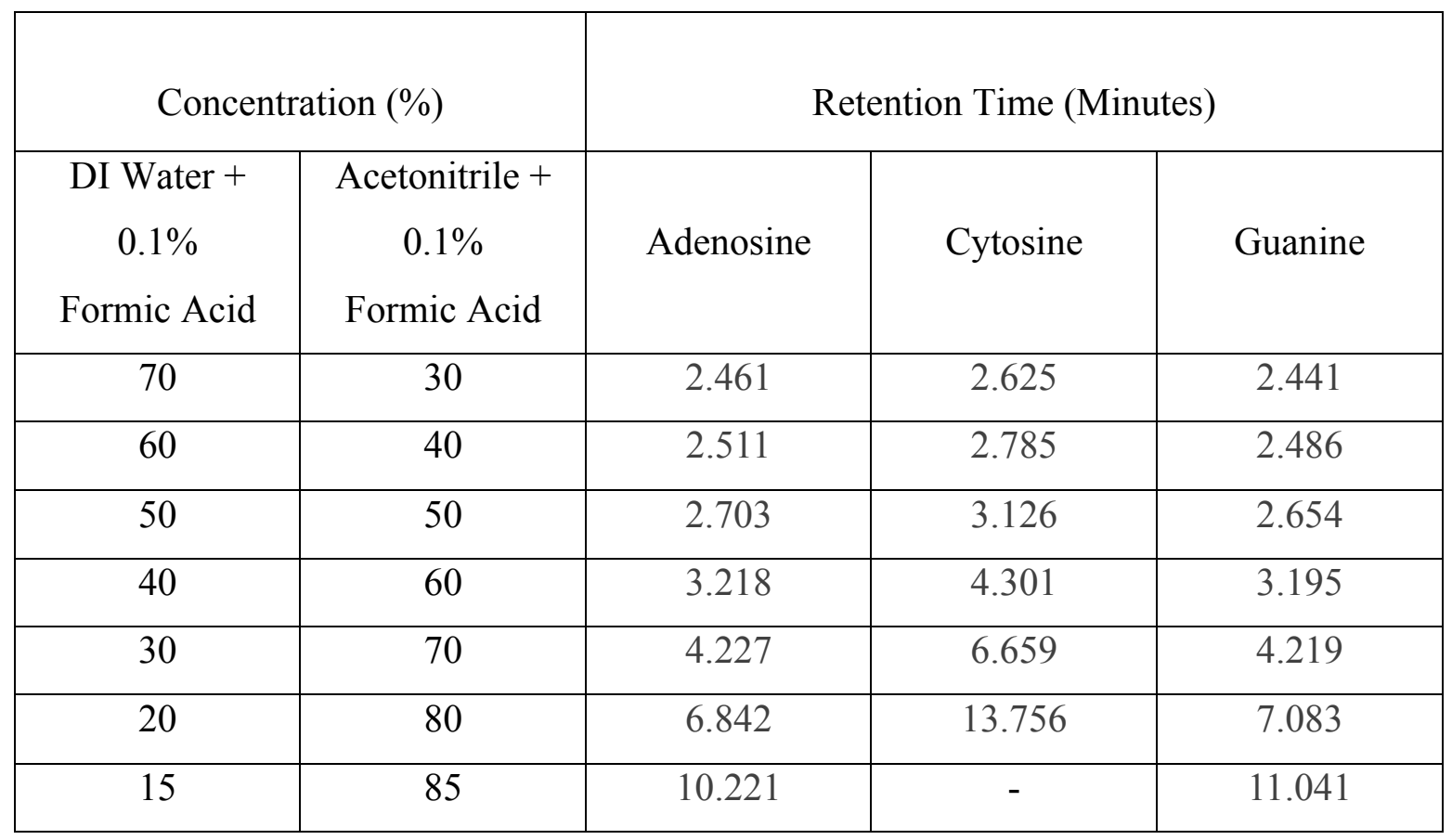

v

Table 8: Retention Time for Thymine and Uracil.

\begin{tabular}{|c|c|c|c|}
\hline \multicolumn{2}{|c|}{ Concentration (\%) } & \multicolumn{2}{c|}{ Retention Time (Minutes) } \\
\hline $\begin{array}{c}\text { DI Water }+ \\
0.1 \%\end{array}$ & Acetonitrile + & Thymine & Uracil \\
Formic Acid & Formic Acid & & \\
\hline 70 & 30 & 2.395 & 2.272 \\
\hline 60 & 40 & 2.271 & 2.199 \\
\hline 50 & 50 & 2.224 & 2.166 \\
\hline 40 & 60 & 2.236 & 2.191 \\
\hline 30 & 70 & 2.328 & 2.312 \\
\hline 20 & 80 & 2.488 & 2.515 \\
\hline 15 & 85 & 2.729 & 2.741 \\
\hline
\end{tabular}


Figure 18 shows the retention pattern for adenosine, cytosine, and guanine. There is a consistent increase in the retention time for these three compounds with an increase in the $\% \mathrm{~B}$ in the mobile phase. The typical ANP retention pattern is clearly observed. At the low $\mathrm{pH}$ values, all these nucleobase compounds should be in a positively charged ionic form.

Figure 19 depicts the retention profile for uracil and thymine. Uracil is a highly polar compound. Because of its high polarity and non-retentive tendency, uracil is used to determine void volume in reverse phase mode chromatography. However, uracil does exhibit significant retention on the silica hydride-based stationary phase. Although guanine and uracil show the least retention among all nucleobase test compounds, there is a distinguishable increase in the retention time with an increase in the $\% \mathrm{~B}$ in the mobile phase. 


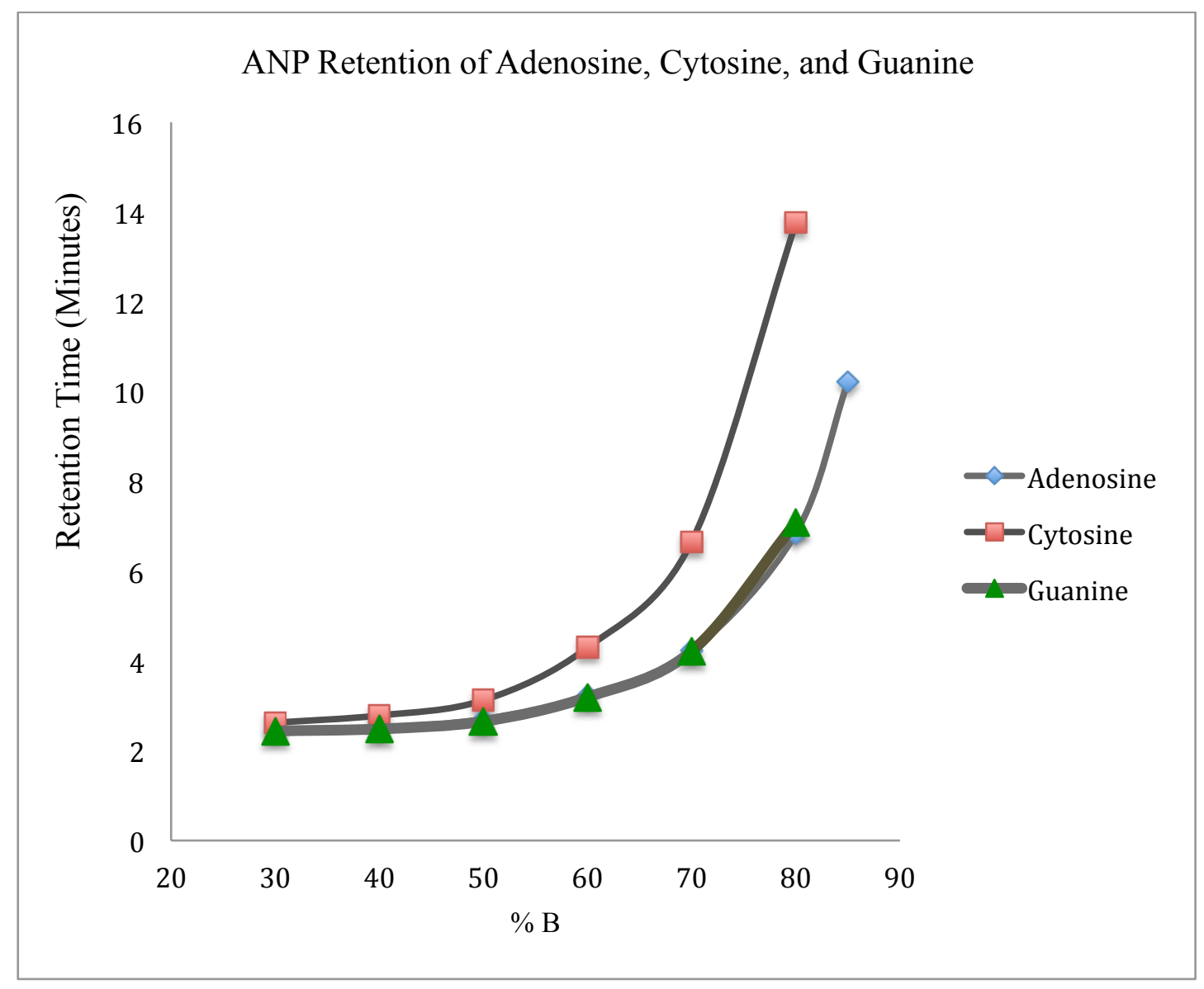

Figure 18: ANP Retention of Adenosine, Cytosine, and Guanine. 


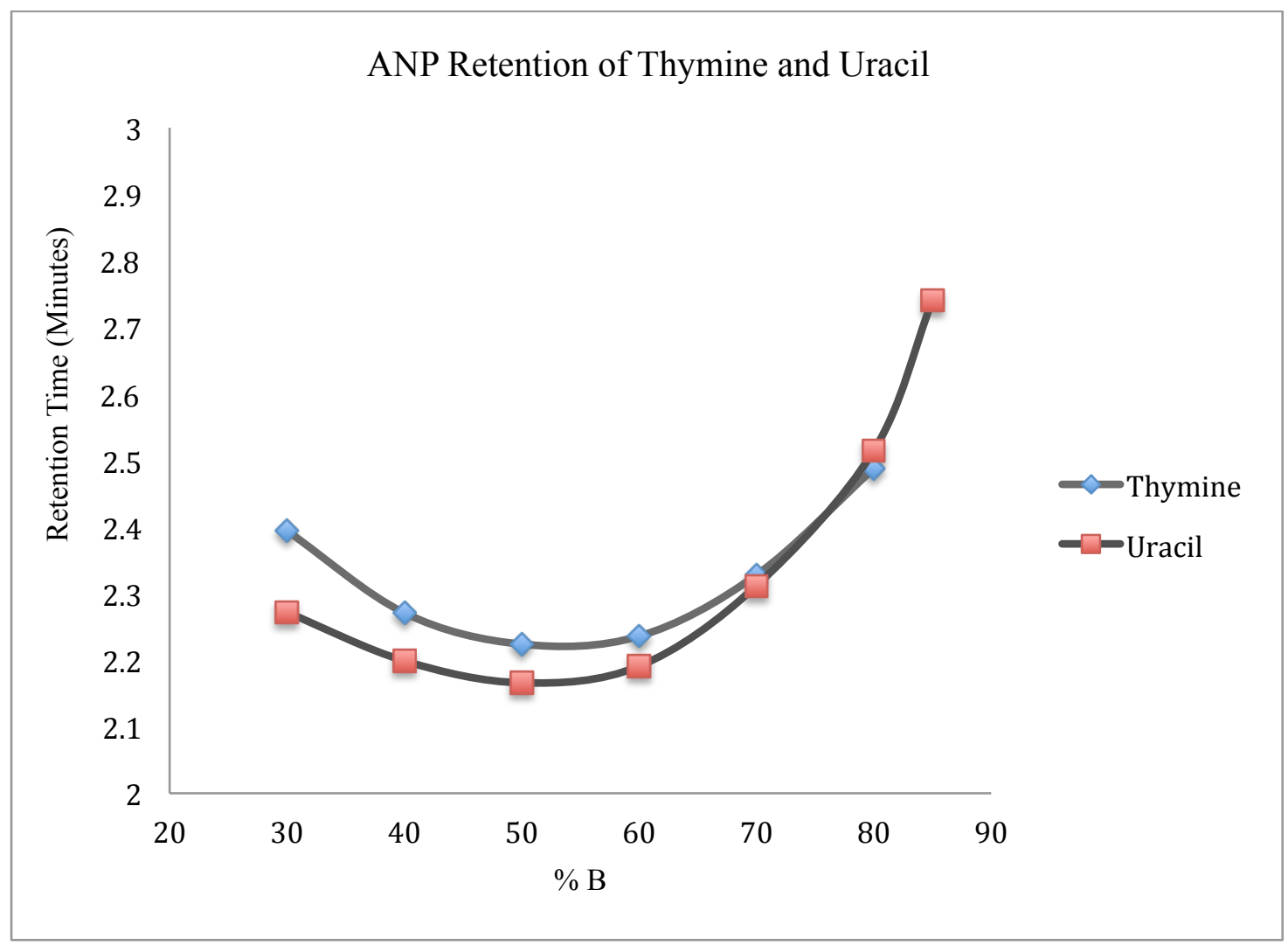

Figure 19: ANP Retention of Thymine and Uracil.

\subsection{ANP Retention of Miscellaneous Compounds}

Three miscellaneous compounds studied for ANP retention were creatine hydrate, creatinine, and thiamine hydrochloride. Creatine hydrate and creatinine are ergogenic acids, which are polar in nature. Both these samples were dissolved in 50:50 A:B with $0.1 \%$ formic acid. Retention time was recorded as a function of varying concentration of solvent B. The optimized injection volume was $5 \mu \mathrm{L}$. The flow rate was $0.5 \mathrm{~mL} /$ minute. The UV detector was used and the selected wavelength of detection was $210 \mathrm{~nm}$. Table 9 shows the retention times for creatine hydrate and creatinine at different compositions of the mobile phase. 
Thiamine also was dissolved in 50:50 A:B with $0.1 \%$ formic acid. Retention time was recorded as a function of varying concentration solvent $\mathrm{B}$. The optimized injection volume was $5 \mu \mathrm{L}$. The flow rate was $0.5 \mathrm{~mL} /$ minute. The UV detector was used with the selected wavelength of detection as $254 \mathrm{~nm}$. Table 10 lists the retention time for thiamine at varying compositions of solvent $\mathrm{A}$ and solvent $\mathrm{B}$.

Figure 20 shows that creatine hydrate and creatinine are very well retained in the ANP mode on perfluorinated silica hydride based stationary phase. It is possible that the perfluorinated moiety plays an important role in the retention of these two compounds, whereas the hydride part provides selectivity [25]. Thiamine shows remarkable retention with retention times unusually long. Figure 21 shows the plot of retention time in minutes for thiamine as a function of $\% \mathrm{~B}$.

All these polar compounds have successfully demonstrated the aqueous normal phase type of retention on the perfluorinated silica hydride-based stationary phase. It is most likely that the combined effect of the perfluorinated moiety and silica hydride functionality play a significant role in their ANP retention behavior. 
Table 9: Retention Time for Creatine Hydrate and Creatinine.

\begin{tabular}{|c|c|c|c|}
\hline \multicolumn{2}{|c|}{ Concentration (\%) } & \multicolumn{2}{c|}{ Retention Time (Minutes) } \\
\hline $\begin{array}{c}\text { DI Water }+ \\
0.1 \% \\
\text { Formic Acid }\end{array}$ & $\begin{array}{c}\text { Acetonitrile } \\
0.1 \% \\
\text { Formic Acid }\end{array}$ & Creatine Hydrate & Creatinine \\
\hline 70 & 30 & 3.098 & 3.276 \\
\hline 60 & 40 & 3.109 & 3.256 \\
\hline 50 & 50 & 3.717 & 3.434 \\
\hline 40 & 60 & 4.88 & 4.215 \\
\hline 30 & 70 & 7.53 & 5.739 \\
\hline 20 & 80 & 15.34 & 9.841 \\
\hline 15 & 85 & - & 14.412 \\
\hline
\end{tabular}




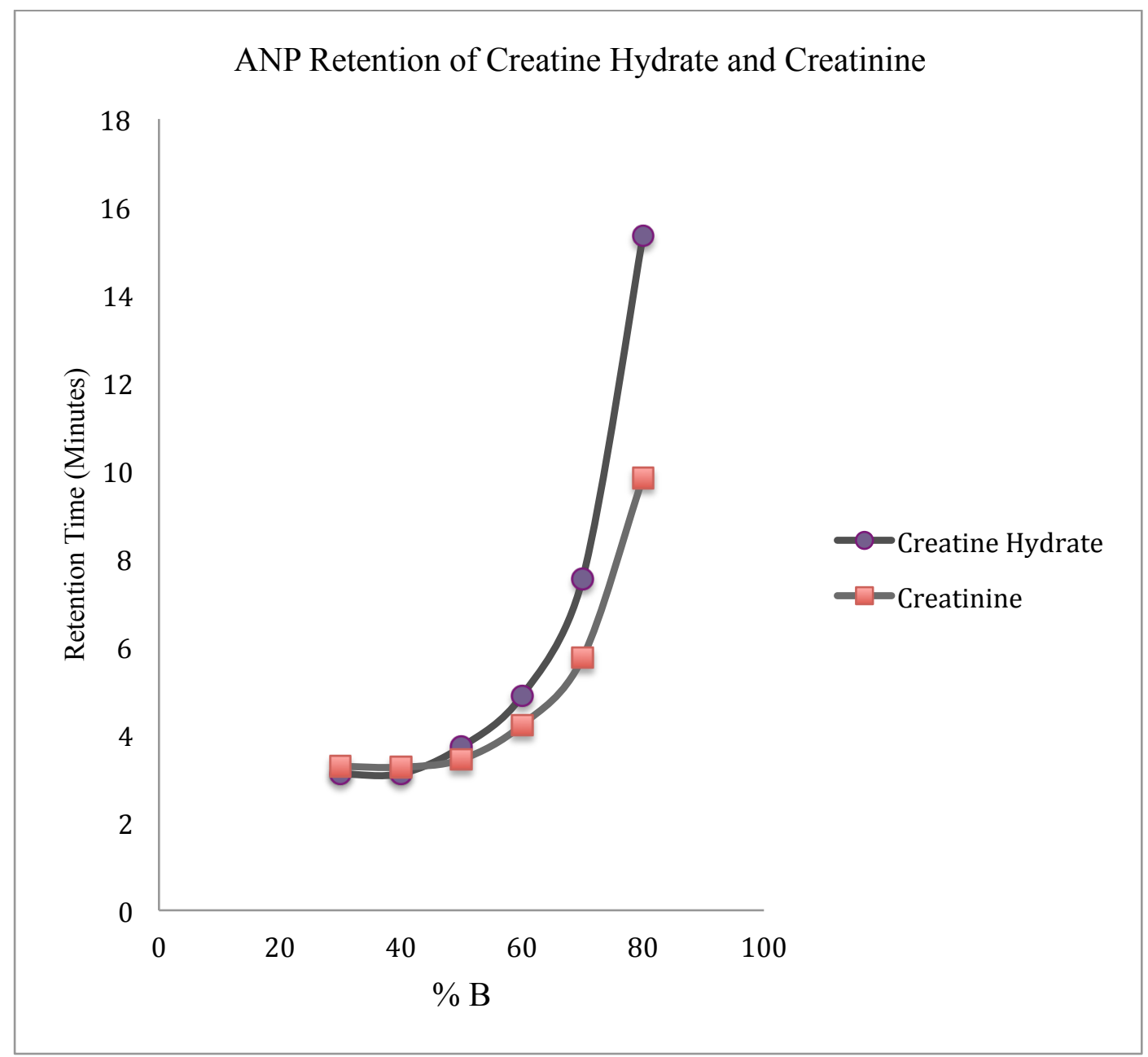

Figure 20: ANP Retention of Creatine Hydrate and Creatinine 
Table 10: Retention Time for Thiamine.

\begin{tabular}{|c|c|c|}
\hline \multicolumn{2}{|c|}{ Concentration (\%) } & \multirow{2}{*}{$\begin{array}{c}\text { Retention Time (Minutes) } \\
\text { Thiamine }\end{array}$} \\
\hline $\begin{array}{l}\text { DI Water + } \\
0.1 \% \\
\text { Formic Acid }\end{array}$ & $\begin{array}{c}\text { Acetonitrile }+ \\
0.1 \% \\
\text { Formic Acid }\end{array}$ & \\
\hline 80 & 20 & 3.426 \\
\hline 75 & 25 & 3.54 \\
\hline 70 & 30 & 3.58 \\
\hline 65 & 35 & 4.06 \\
\hline 60 & 40 & 5.24 \\
\hline 55 & 45 & 6.118 \\
\hline 50 & 50 & 8.51 \\
\hline 45 & 55 & 12.146 \\
\hline 40 & 60 & 16.9 \\
\hline 35 & 65 & 25.86 \\
\hline
\end{tabular}




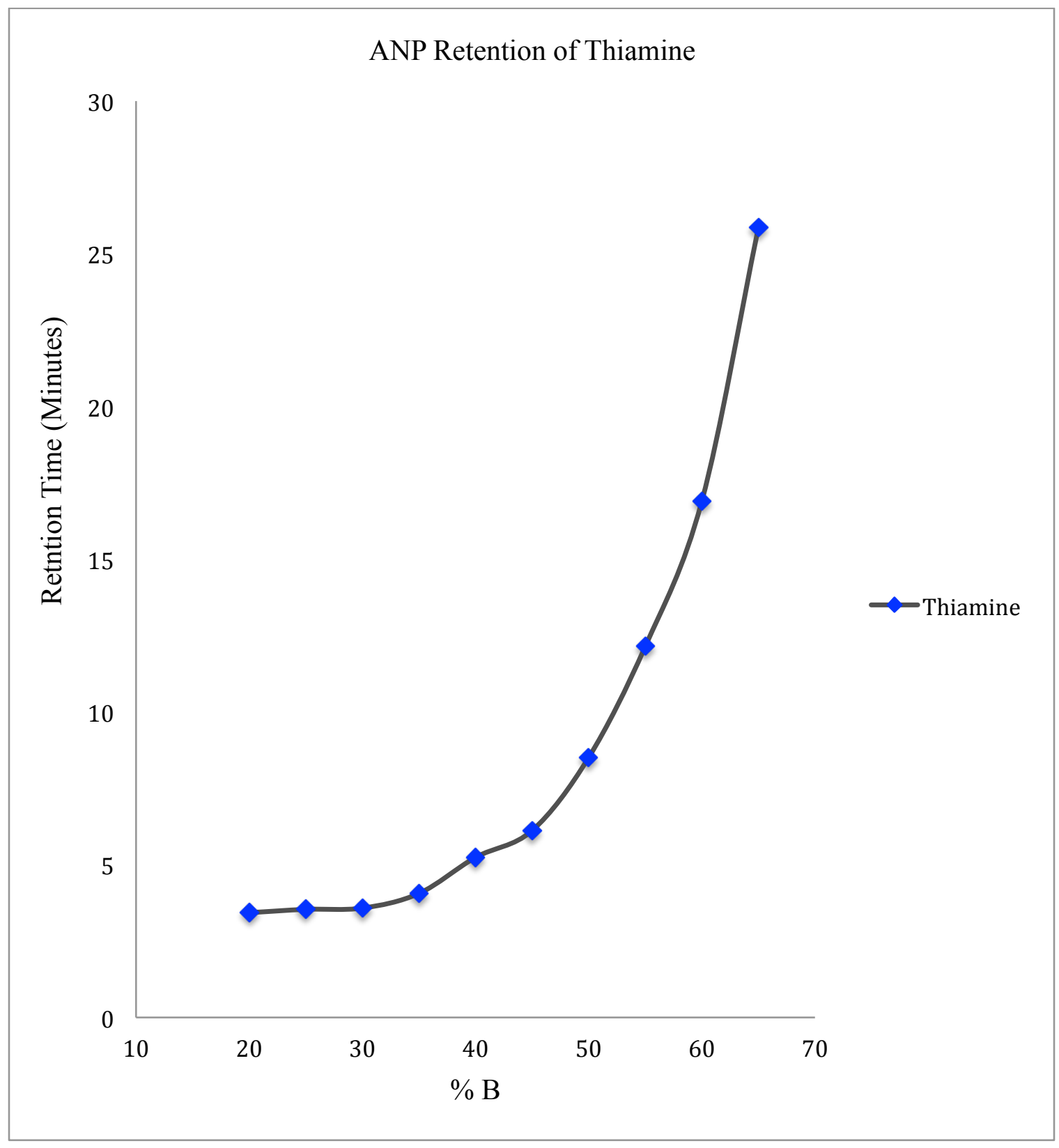

Figure 21: ANP Retention of Thiamine. 


\section{RP Retention Profile}

For the perfluorinated silica hydride-based stationary phase, the ANP retention behavior of a wide variety of polar compounds was very well established. The next step of this project was to test retention of nonpolar samples in the reverse phase mode. Polycyclic aromatic hydrocarbons (PAHs) were used as test compounds. Fluorene, pyrene, naphthalene, and phenanthrene were dissolved in 100:0 acetonitrile with $0.1 \%$ formic acid so as to have sample concentration $1 \mathrm{mg} / \mathrm{mL}$. Retention times were recorded as a function of varying concentrations of solvent $\mathrm{B}$. The optimized injection volume was set at $4 \mu \mathrm{L}$ with the flow rate at $0.5 \mathrm{~mL} /$ minute. The $\mathrm{UV}$ detector was used and selected wavelength of detection was $254 \mathrm{~nm}$. Table 11 shows the retention times for fluorene, pyrene, naphthalene, and phenanthrene at different compositions of the mobile phase.

Figure 22 depicts the classic reverse phase retention maps for four PAHs. The higher the percent of polar solvent (DI water) in the mobile phase, the higher retention time for all these nonpolar analytes. On the contrary, with an increase in the percent of nonpolar solvent (acetonitrile), there was a significant decrease in the retention time. Above $70 \%$ of acetonitrile, retention times are almost constant. It should be noted that the void volume of the column was $1.7 \mathrm{~mL}$; hence, for PAHs even at a higher percent of acetonitrile $(80 \%, 90 \%)$, there is some retention at around 2.3 minutes. This result provided sufficient evidence to prove that the silica hydride-based perfluorinated stationary phase can operate in reverse phase conditions. 
Table 11: Retention Time for Fluorene, Pyrene, Naphthalene, and Phenanthrene.

\begin{tabular}{|c|c|c|c|c|c|}
\hline \multicolumn{2}{|c|}{ Concentration (\%) } & \multicolumn{3}{|c|}{ Retention Time (Minutes) } & Phenanthrene \\
\hline $\begin{array}{c}\text { DI Water }+ \\
0.1 \% \\
\text { Formic Acid } \\
\text { Formic Acid }\end{array}$ & $\begin{array}{c}\text { Acetonitrile } \\
0.1 \%\end{array}$ & Pyrene & Naphthalene & Fluorene & Phe \\
\hline 70 & 30 & 12.71 & 8.429 & 9.056 & 10.15 \\
\hline 60 & 40 & 5.742 & 4.291 & 5.083 & 5.261 \\
\hline 50 & 50 & 3.633 & 3.202 & 3.427 & 3.465 \\
\hline 40 & 60 & 2.672 & 2.62 & 2.623 & 2.632 \\
\hline 30 & 70 & 2.35 & 2.422 & 2.403 & 2.413 \\
\hline 20 & 80 & 2.287 & 2.322 & 2.289 & 2.323 \\
\hline 10 & 90 & 2.317 & 2.331 & 2.326 & 2.325 \\
\hline
\end{tabular}




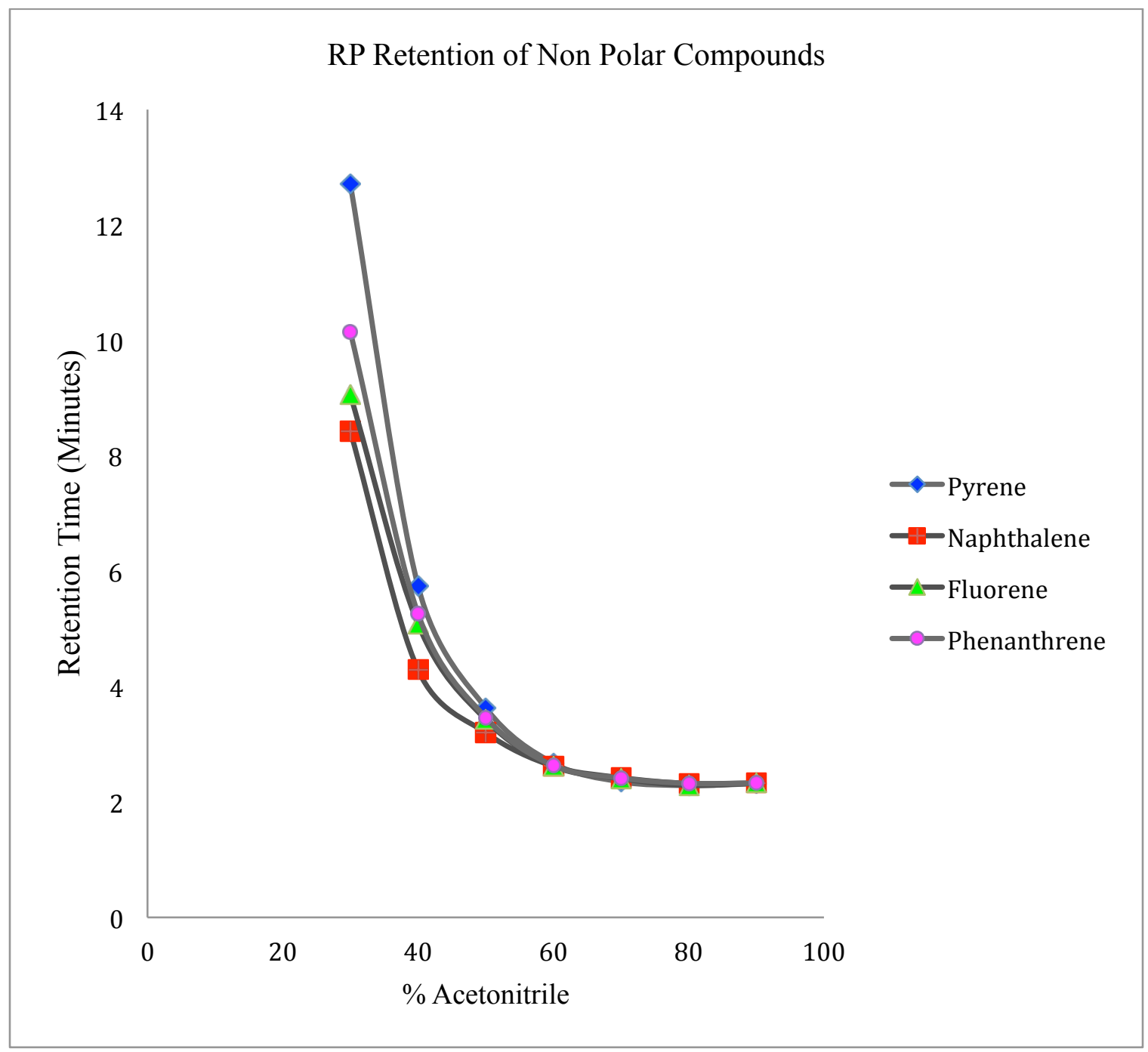

Figure 22: RP Retention of Pyrene, Naphthalene, Fluorine, and Phenanthrene. 


\section{U-shaped Retention Profile}

Figure 23 shows a $\mathrm{U}$-shaped retention profile for the polar and nonpolar compounds. The right side of the curve represents ANP retention of polar compounds, and the left side of the curve represents the RP retention of nonpolar compounds. The versatility of this stationary phase to operate in reverse phase and normal phase conditions was well established.

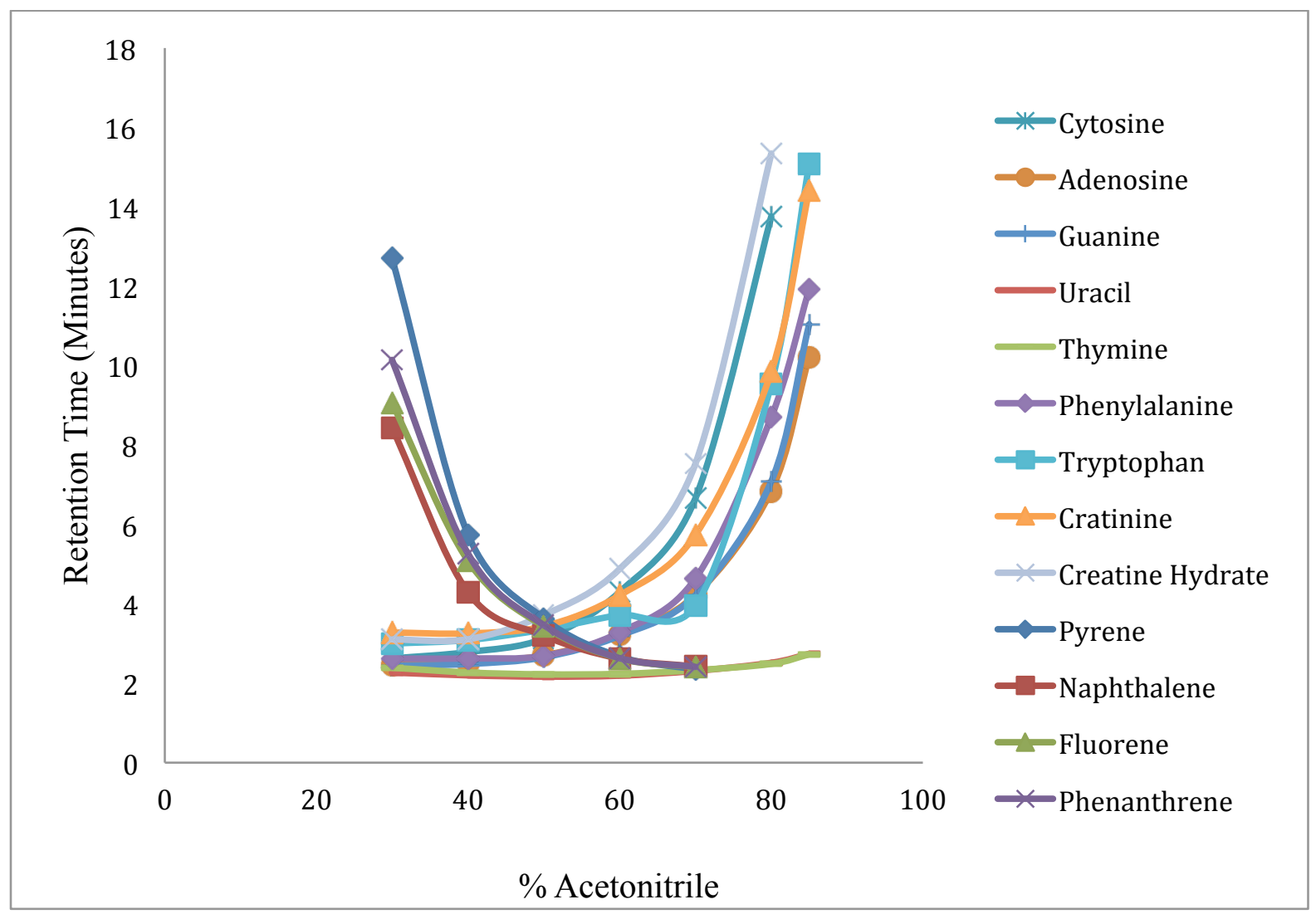

Figure 23: U-shaped Retention Profile for Perfluorinated Silica Hydride Stationary Phase

The initial sets of experiments helped to achieve one of the important goals of this project. The dual retention ability of the perfluorinated silica hydride-based stationary phase was confirmed. 


\section{E. pH Studies with Different Buffer Systems}

The next set of experiments was designed to study the effect of varying concentrations

of buffer on the retention time of the polar and nonpolar solutes. Two buffer systems with different $\mathrm{pH}$ ranges used were formic acid and ammonium acetate buffer. The retention time for different test compounds was recorded as a function of concentration of buffer. Because the upper limit for silica hydride-based columns is around $\mathrm{pH} 7$, lower $\mathrm{pH}$ ranges were selected for this study.

\section{Formic Acid Buffer System}

Formic acid is the most compatible buffer system with silica hydride-based stationary phases. To study the effect of $\mathrm{pH}$ different concentrations of formic acids prepared were: $0.05 \%, 0.075 \%, 0.1 \%, 0.2 \%$, and $0.3 \%$. DI Water was used as solvent $\mathrm{A}$ and acetonitrile was used as solvent B. A mobile phase composition of 20:80 A:B was used with five different percentages of the formic acid. Polar test compounds used to study the effect of $\mathrm{pH}$ were adenosine, guanine, tryptophan, and creatine hydrate. Five test samples were prepared for every single compound with five different buffer concentrations. Toluene was used as a neutral test compound. The UV detector was used for this analysis and detection wavelength was set to $254 \mathrm{~nm}$. The optimized sample injection volume was 5 $\mu \mathrm{L}$ with the flow rate of $0.5 \mathrm{~mL} /$ minute. The $\mathrm{pH}$ of the $20: 80$ composition of $\mathrm{A}: \mathrm{B}$ solution at five different buffer concentrations was measured using a Fisher Scientific Accumet $\mathrm{pH}$ meter. The $\mathrm{pH}$ meter was two point calibrated at $\mathrm{pH} 4.0$ and 7.0. 
Table 12 lists the $\mathrm{pH}$ values at 20:80 composition of $\mathrm{A}: \mathrm{B}$ at five different concentrations of formic acid. Table 13 summarizes retention times in minutes for four polar and one nonpolar test compounds.

Table 12: pH values for Different Concentrations of Formic Acids.

\begin{tabular}{|c|c|}
\hline \multicolumn{2}{|c|}{ Water + Acetonitrile (20:80) } \\
\hline \% Formic Acid & $\mathrm{pH}$ \\
\hline 0.05 & 3.02 \\
\hline 0.075 & 2.98 \\
\hline 0.1 & 2.93 \\
\hline 0.2 & 2.89 \\
\hline 0.3 & 2.86 \\
\hline
\end{tabular}

Table 13: Retention Time (Minutes) at Different Concentrations of Formic Acid.

\begin{tabular}{|c|c|c|c|c|c|}
\hline \% Formic Acid & Adenosine & Guanine & Tryptophan & $\begin{array}{c}\text { Creatine } \\
\text { Hydrate }\end{array}$ & Toluene \\
\hline 0.05 & 6.587 & 6.495 & 10.11 & 6.989 & 2.087 \\
\hline 0.075 & 7.124 & 5.804 & 8.799 & 6.94 & 2.018 \\
\hline 0.1 & 6.671 & 6.473 & 8.917 & 6.561 & 2.014 \\
\hline 0.2 & 6.54 & 5.843 & 7.572 & 7.103 & 2.011 \\
\hline 0.3 & 6.00 & 5.712 & 6.76 & 5.41 & 2.004 \\
\hline
\end{tabular}



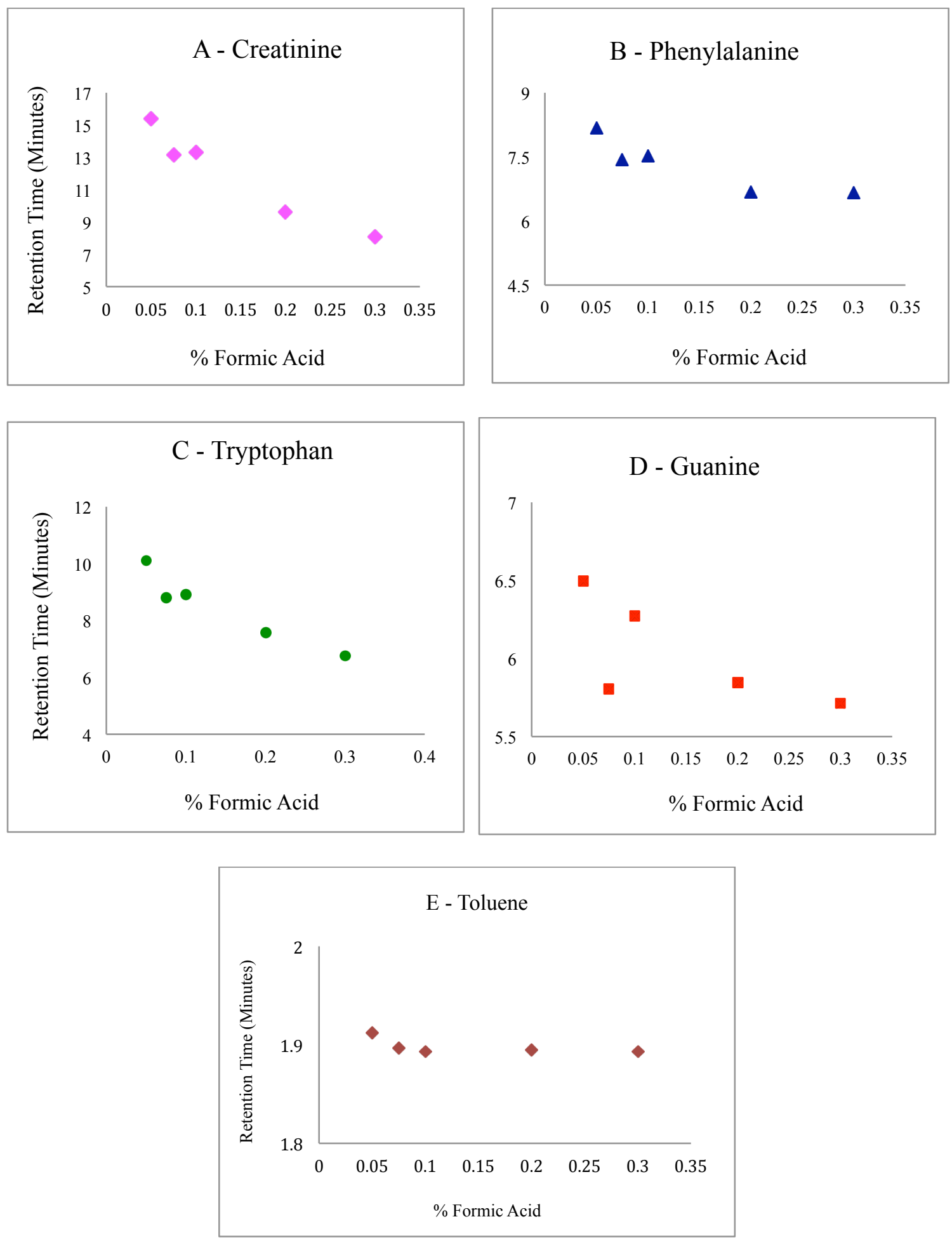

Figure 24(A-E): Retention Time as a Function of Concentration of Formic Acid. 
Figure 24 (A-E) includes five different plots for creatinine, phenylalanine, tryptophan, guanine, and toluene. It shows the plot of retention time as a function of the percent of formic acid for five different solutes. The first four plots (A-D) account for polar compounds and plot $\mathrm{E}$ for neutral compound toluene. All four polar test compounds show a similar trend of retention times with varying buffer concentration. With an increase in the concentration of buffer formic acid from $0.05 \%$ to $3 \%$, there is a decrease in the retention time of polar solutes. However, in the case of a neutral compound toluene (figure 24-E), there is no apparent change in the retention time with varying concentrations of the buffer.

\section{Ammonium Acetate Buffer System}

The next buffer system used was ammonium acetate buffer. Ammonium acetate buffer had higher $\mathrm{pH}$ compared to formic acid. Two hundred and fifty $\mathrm{mL}$ of $300 \mathrm{mM}$ ammonium acetate stock solution was prepared by dissolving $5.7811 \mathrm{~g}$ of ammonium acetate in $250 \mathrm{~mL}$ of DI water. Three hundred $\mathrm{mM}$ stock solution was used to prepare a set of polar (DI water) and nonpolar (acetonitrile) solvents with varying concentrations of ammonium acetate. Different buffer concentrations used for this study were $2 \mathrm{mM}, 4$ $\mathrm{mM}, 6 \mathrm{mM}, 8 \mathrm{mM}$, and $10 \mathrm{mM}$. To make $500 \mathrm{~mL}$ of $2 \mathrm{mM}$ DI water solvent, a calculated amount of $300 \mathrm{mM}$ stock was simply added to DI water. Likewise, five different concentrations of solvent A (DI water) were prepared. Preparation of nonpolar solvent B (acetonitrile) needed extra care because ammonium acetate does not dissolve in acetonitrile. Hence, there has to be some amount of water in the acetonitrile for it to dissolve the required amount of ammonium acetate solution. A calculated amount of 300 
$\mathrm{mM}$ ammonium acetate was added to the acetonitrile, which had $25 \% \mathrm{v} / \mathrm{v}$ of DI water. For example, the total volume of $500 \mathrm{~mL}$ of solvent B actually contained $475 \mathrm{~mL}$ acetonitrile and $25 \mathrm{~mL}$ of DI water. The aqueous portion of $25 \mathrm{~mL}$ consisted of the required amount of ammonium acetate and remaining DI water. The actual amount of acetonitrile was taken into consideration while calculating required volumes of buffer solution to be used to prepare five different buffer concentrations.

\subsection{Compatibility of Ammonium Acetate Buffer With Perfluorinated Silica}

Hydride-based Stationary Phase

The compatibility of buffer with silica hydride-based perfluorinated column was first tested by recording retention times for three polar solutes as a function of $\% \mathrm{~B}$ at constant buffer concentration. Test solutes used to check compatibility were phenylalanine, cytosine, and adenosine. Buffer concentration was set to $4 \mathrm{mM}$. The UV detector was used with $254 \mathrm{~nm}$ as a detection wavelength. The optimized sample injection volume was $5 \mu \mathrm{L}$, and the flow rate was set to $0.5 \mathrm{~mL} / \mathrm{minute}$.

Table 14 lists the retention times for three test solutes in minutes at different compositions of the mobile phase. Figure 25 shows a retention map for three solutes: phenylalanine, cytosine, and adenosine, as a function of $\% \mathrm{~B}$ with $4 \mathrm{mM}$ buffer concentration. Figure 25 depicts that three polar test solutes exhibit typical ANP retention profile in the presence of ammonium acetate buffer. Therefore, it was concluded that ammonium acetate is a suitable buffer system for the perfluorinated silica hydride based stationary phase. 
Table 14: Retention Times for Polar Solutes with Ammonium Acetate Buffer.

\begin{tabular}{|c|c|c|c|c|}
\hline \multicolumn{2}{|c|}{} & \multicolumn{3}{|c|}{ Retention Time (Minutes) } \\
\hline $\begin{array}{c}\text { DI Water }+ \\
4 \mathrm{mM} \\
\text { Ammonium } \\
\text { Acetate }\end{array}$ & $\begin{array}{c}\text { Acetonitrile } \\
+ \\
4 \mathrm{mM} \\
\text { Ammonium } \\
\text { Acetate }\end{array}$ & Phenylalanine & Cytosine & Adenosine \\
\hline 50 & 50 & 2.108 & 2.15 & 1.961 \\
\hline 40 & 60 & 2.18 & 2.184 & 1.976 \\
\hline 35 & 65 & 2.406 & 2.264 & 2.039 \\
\hline 30 & 70 & 2.621 & 2.421 & 2.042 \\
\hline 25 & 75 & 2.961 & 2.546 & 2.131 \\
\hline 20 & 80 & 3.565 & 3.038 & 2.462 \\
\hline
\end{tabular}




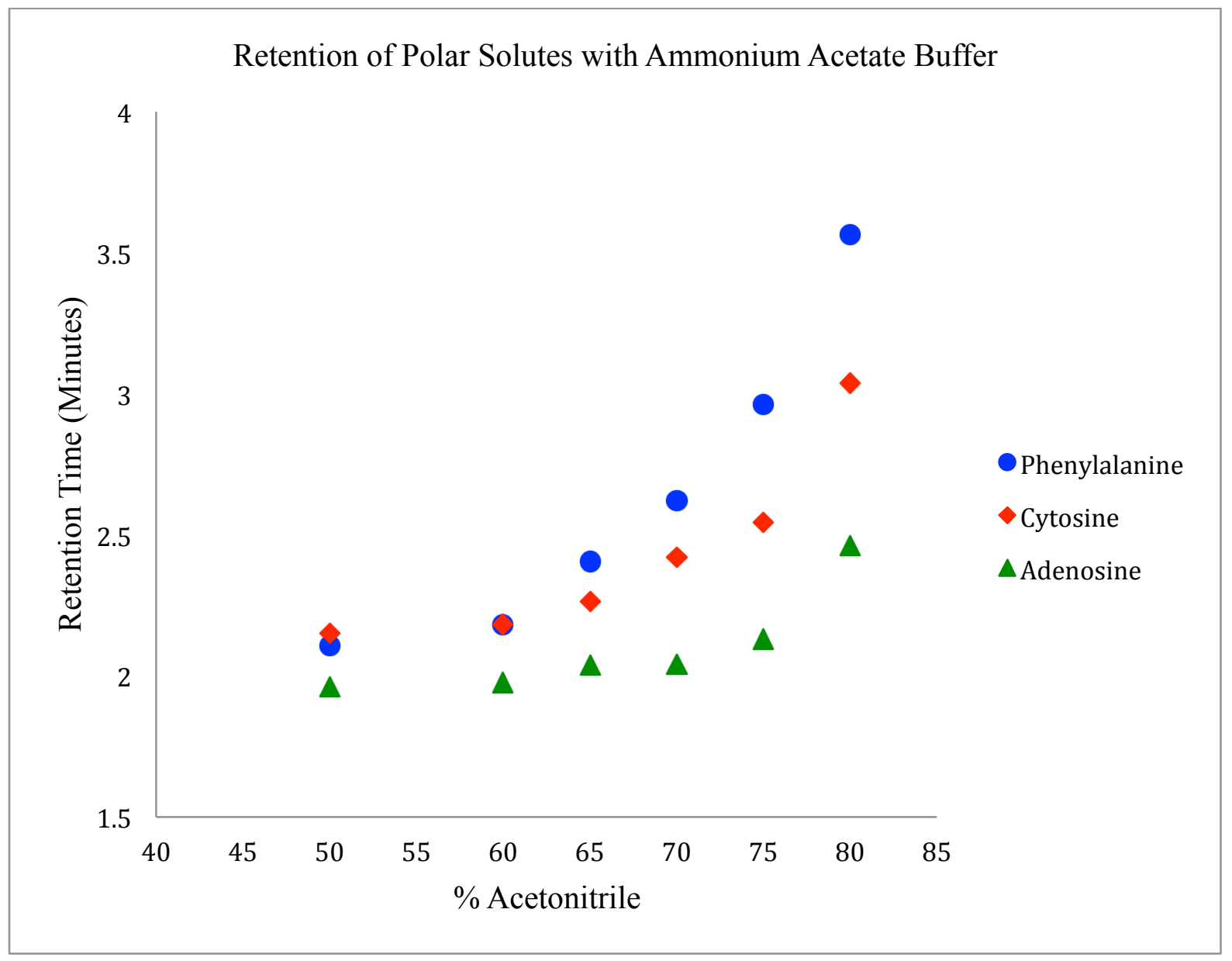

Figure 25: Retention of Polar Solutes with Ammonium Acetate Buffer. 


\subsection{Varying Concentration of Ammonium Acetate Buffer}

Once the compatibility of the ammonium acetate buffer system was established, the next step was to study the effects of varying concentrations of the buffer. As described in the previous section, solvents $\mathrm{A}$ and $\mathrm{B}$ with five different concentrations of ammonium acetate were prepared. The 20:80 A:B composition was used for all the retention time measurements. Polar test compounds used were phenylalanine, cytosine, and adenosine. Toluene was used as a nonpolar test compound. The $\mathrm{pH}$ of the 20:80 composition of A:B solution at five different buffer concentrations was measured using Fisher Scientific Accumet $\mathrm{pH}$ meter. The $\mathrm{pH}$ meter was two point calibrated at $\mathrm{pH} 4.0$ and 7.0. The UV detector was used for this analysis and the detection wavelength was $254 \mathrm{~nm}$. The optimized sample injection volume was $5 \mu \mathrm{L}$. The flow rate was set to $0.5 \mathrm{~mL} /$ minute.

Table 15 lists the $\mathrm{pH}$ values at 20:80 composition of $\mathrm{A}: \mathrm{B}$ and five different concentrations of ammonium acetate buffer. Table 16 summarizes retention times in minutes for four polar and one nonpolar compounds.

Figure $26(\mathrm{~A}-\mathrm{D})$ includes four different plots for phenylalanine, cytosine, adenosine, and toluene. It shows the plot of retention time as a function of concentration of ammonium acetate $(\mathrm{mM})$ for four different solutes. The first three plots $(A-C)$ account for polar compounds and a plot D for a neutral compound, toluene. A similar trend in the retention times for polar compounds with increase in buffer concentration can be easily identified. With an increase in the concentration of buffer ammonium acetate from $2 \mathrm{mM}$ to $10 \mathrm{mM}$, there is a decrease in the retention time of polar solutes. However, in the case 
of neutral compound toluene (figure 26-D), there is no apparent change in the retention time with varying concentration of the buffer.

Table 15: pH values for Different Concentrations of Ammonium Acetate Buffer.

\begin{tabular}{|c|c|}
\hline \multicolumn{2}{|c|}{ Water + Acetonitrile $(20: 80)$} \\
\hline $\begin{array}{c}\text { Concentration of } \\
\text { Ammonium } \\
\text { Acetate }(\mathrm{mM})\end{array}$ & $\mathrm{pH}$ \\
\hline 2 & 6.13 \\
\hline 4 & 5.98 \\
\hline 6 & 5.87 \\
\hline 8 & 5.72 \\
\hline 10 & 5.61 \\
\hline
\end{tabular}

Table 16: Retention Time at Different Concentrations of Ammonium Acetate Buffer.

\begin{tabular}{|c|c|c|c|c|}
\hline \multirow{2}{*}{$\begin{array}{c}\text { Concentration } \\
\text { of Ammonium } \\
\text { Acetate }(\mathrm{mM})\end{array}$} & \multicolumn{4}{|c|}{ Retention Time (Minutes) } \\
\cline { 2 - 5 } & Phenylalanine & Cytosine & Adenosine & Toluene \\
\hline 2 & 4.083 & 3.151 & 2.602 & 1.964 \\
\hline 4 & 3.565 & 3.106 & 2.562 & 1.957 \\
\hline 6 & 3.478 & 3.038 & 2.491 & 1.97 \\
\hline 8 & 3.408 & 3.003 & 2.422 & 1.958 \\
\hline 10 & 3.013 & 3.001 & 2.384 & 1.933 \\
\hline
\end{tabular}



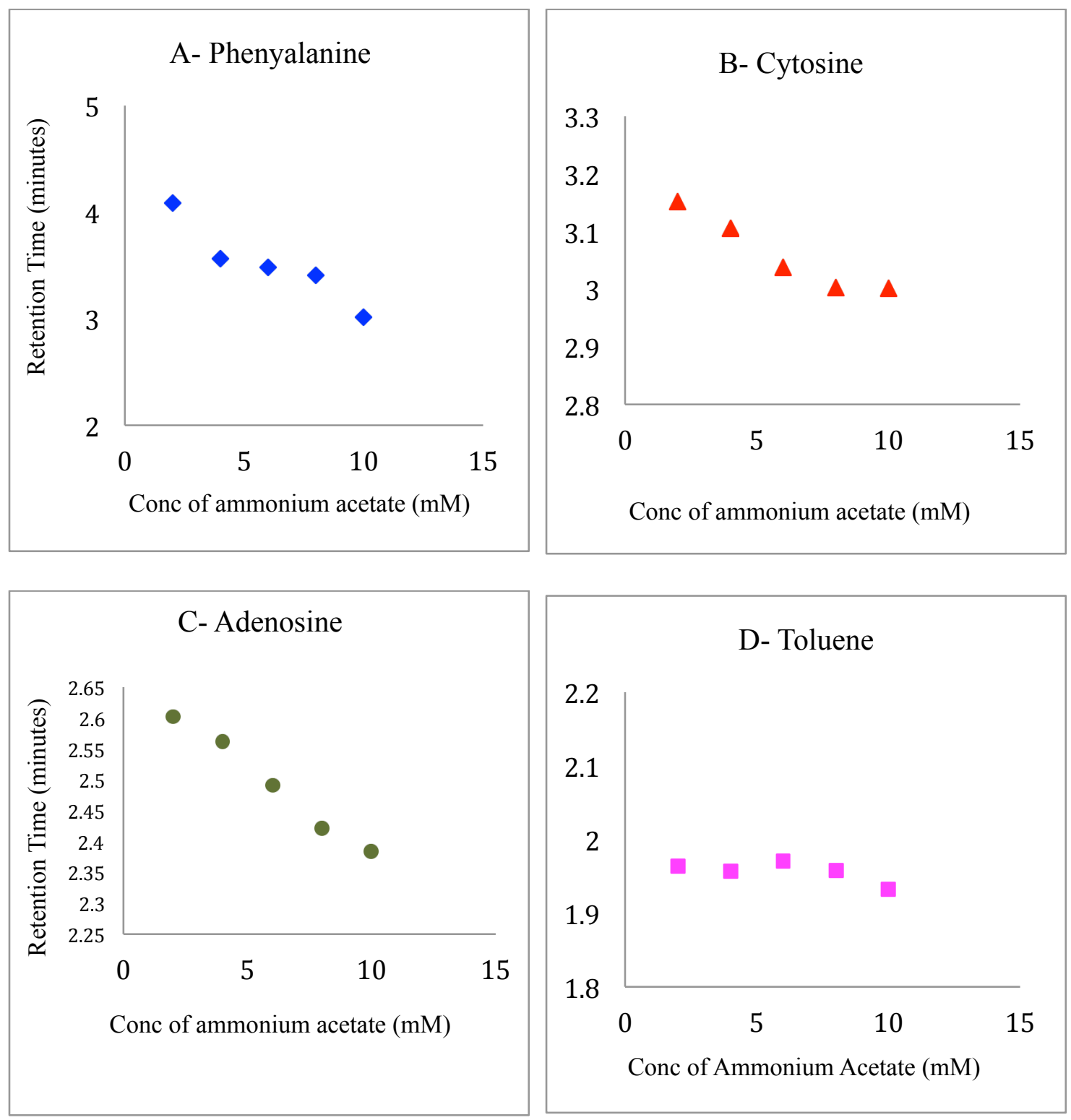

Figure 26(A-D): Retention Time as a Function of Concentration of Ammonium Acetate. 


\section{F. Comparison of Acetone and Acetonitrile as Solvent B}

The last step of this project was to check compatibility of acetone as solvent B (nonpolar solvent) in the mobile phase. As discussed in the previous section, acetone has many advantages over acetonitrile and could be a good alternative to nonpolar acetonitrile. Since acetone has a high UV cutoff around $330 \mathrm{~nm}$, it has limited applications with UV detection. It can be used with the UV detector only when a solute has a wavelength of absorption higher than $330 \mathrm{~nm}$. In this study, all polar test compounds had maximum absorption near $254 \mathrm{~nm}$; therefore, the UV detector could not be used. The Agilent 1100 series (TOF) LC-MS was used for the acetone study.

The focus of this set of experiments was to compare retention data for acetonitrile and acetone. Five hundred $\mathrm{mL}$ of solvent $\mathrm{A}$ (DI water) and B (acetone MS grade) were prepared separately, and $0.1 \% \mathrm{v} / \mathrm{v}$ formic acid was added as buffer to both these solvents. Test compounds used were: L- tryptophan and L-phenylalanine. Samples were prepared by dissolving $1 \mathrm{mg}$ of the compound in $1 \mathrm{~mL}$ of 50:50 solvent composition along with $0.1 \%$ formic acid. Amino acids readily dissolved in acetone and water. The flow rate was optimized to $0.7 \mathrm{~mL} /$ minute. A time of flight mass analyzer was used. Retention times were recorded as a function of $\% \mathrm{~B}$.

The retention factors were calculated for all the retention time readings for Ltryptophan and L-phenylalanine with acetone and acetonitrile. Retention factor values were used for comparison between acetonitrile and acetone. The retention factor was calculated using the following formula - 


$$
k=\frac{t_{r}-t_{0}}{t_{0}}
$$

$k-$ Retention Factor

$t_{r}-$ Retention Time

$t_{0}-$ Void Volume

Table 17 summarizes the retention factors of L-phenylalanine for acetone and acetonitrile.

Table 18 summarizes the retention factors of L-tryptophan for acetone and acetonitrile.

Figure 27 shows a plot of retention factors $(k)$ as a function $\% \mathrm{~B}$ for $\mathrm{L}$ - phenylalanine.

Figure 28 shows a plot of retention factors $(k)$ as a function \% B for L - tryptophan. 
Table 17: Retention Factors for L-Phenylalanine.

\begin{tabular}{|c|c|c|c|c|c|}
\hline $\begin{array}{c}\text { \% } \begin{array}{c}\text { W DI } \\
+ \\
0.1 \% \mathrm{FA}\end{array} \\
+\end{array}$ & $\begin{array}{c}\text { Acetonitrile/ } \\
\text { Acetone } \\
+\end{array}$ & $\mathrm{t}_{\mathrm{R}}(\mathrm{min})$ & $k$ & $\mathrm{t}_{\mathrm{R}}(\mathrm{min})$ & $k$ \\
\cline { 3 - 6 } & $0.1 \% \mathrm{FA}$ & Acetonitrile & Acetonitrile & Acetone & Acetone \\
\hline 50 & 50 & 2.692 & 0.6825 & 1.992 & 0.245 \\
\hline 40 & 60 & 3.282 & 1.05125 & 2.707 & 0.691875 \\
\hline 30 & 70 & 4.639 & 1.899375 & 4.083 & 1.551875 \\
\hline 20 & 80 & 8.699 & 4.436875 & 7.185 & 3.490625 \\
\hline
\end{tabular}

Table 18: Retention Factors for L-Tryptophan.

\begin{tabular}{|c|c|c|c|c|c|}
\hline $\begin{array}{c}\text { \% } \begin{array}{c}\text { W DI } \\
\text { Water } \\
+\end{array} \\
0.1 \% \mathrm{FA}\end{array}$ & $\begin{array}{c}\text { Acetonitrile/ } \\
\text { Acetone } \\
+\end{array}$ & $\mathrm{t}_{\mathrm{R}}(\mathrm{min})$ & $k$ & $\mathrm{t}_{\mathrm{R}}(\mathrm{min})$ & $k$ \\
\cline { 3 - 6 } & $0.1 \% \mathrm{FA}$ & Acetonitrile & Acetonitrile & Acetone & Acetone \\
\hline 50 & 50 & 3.353 & 1.0956 & 2.2430 & 0.4019 \\
\hline 40 & 60 & 3.72 & 1.3250 & 2.8560 & 0.7850 \\
\hline 30 & 70 & 3.96 & 1.4750 & 3.4340 & 1.1463 \\
\hline 20 & 80 & 9.541 & 4.9631 & 7.8890 & 3.9306 \\
\hline
\end{tabular}




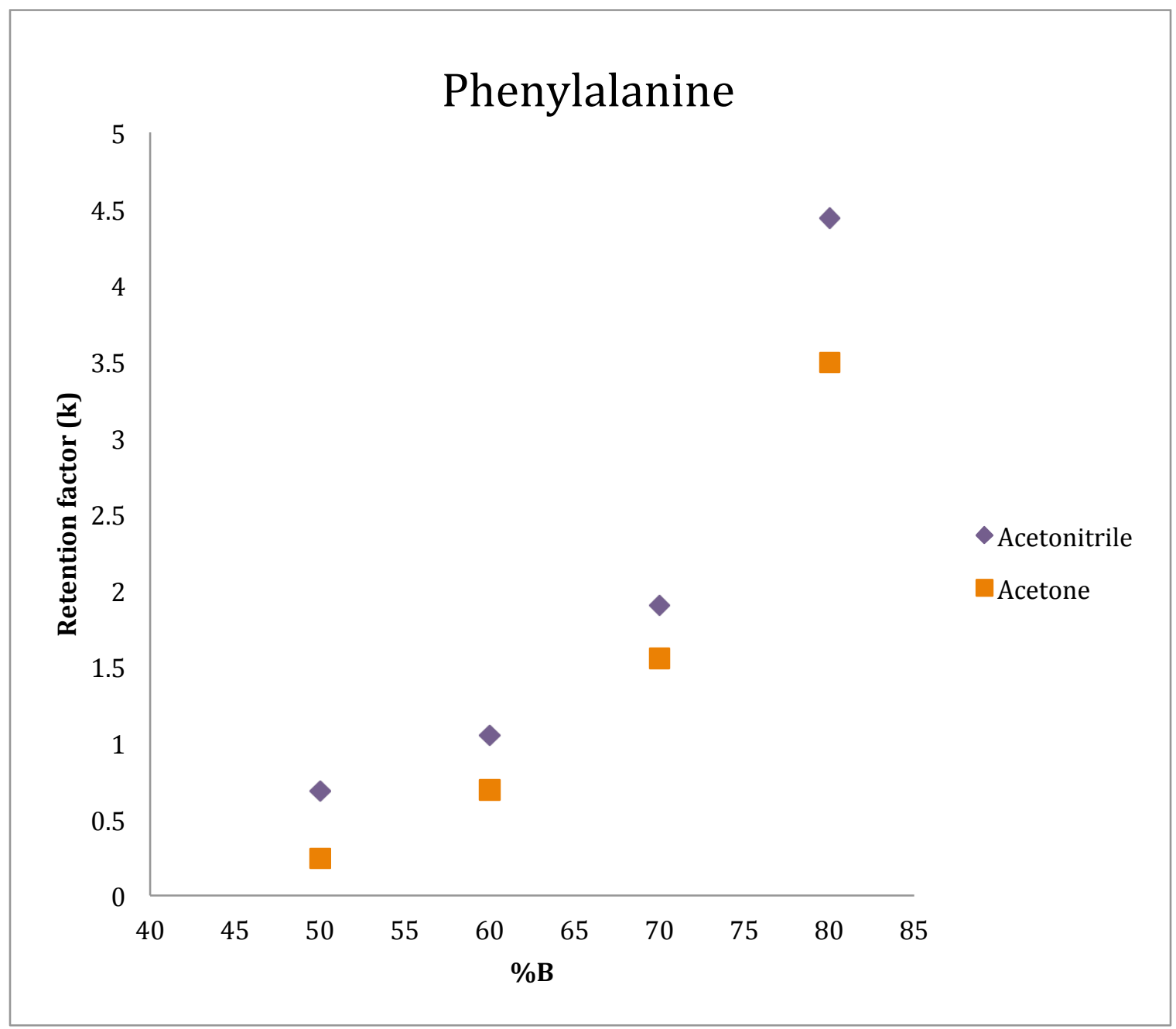

Figure 27: Retention Factor as a Function of \% B for L-phenylalanine. 


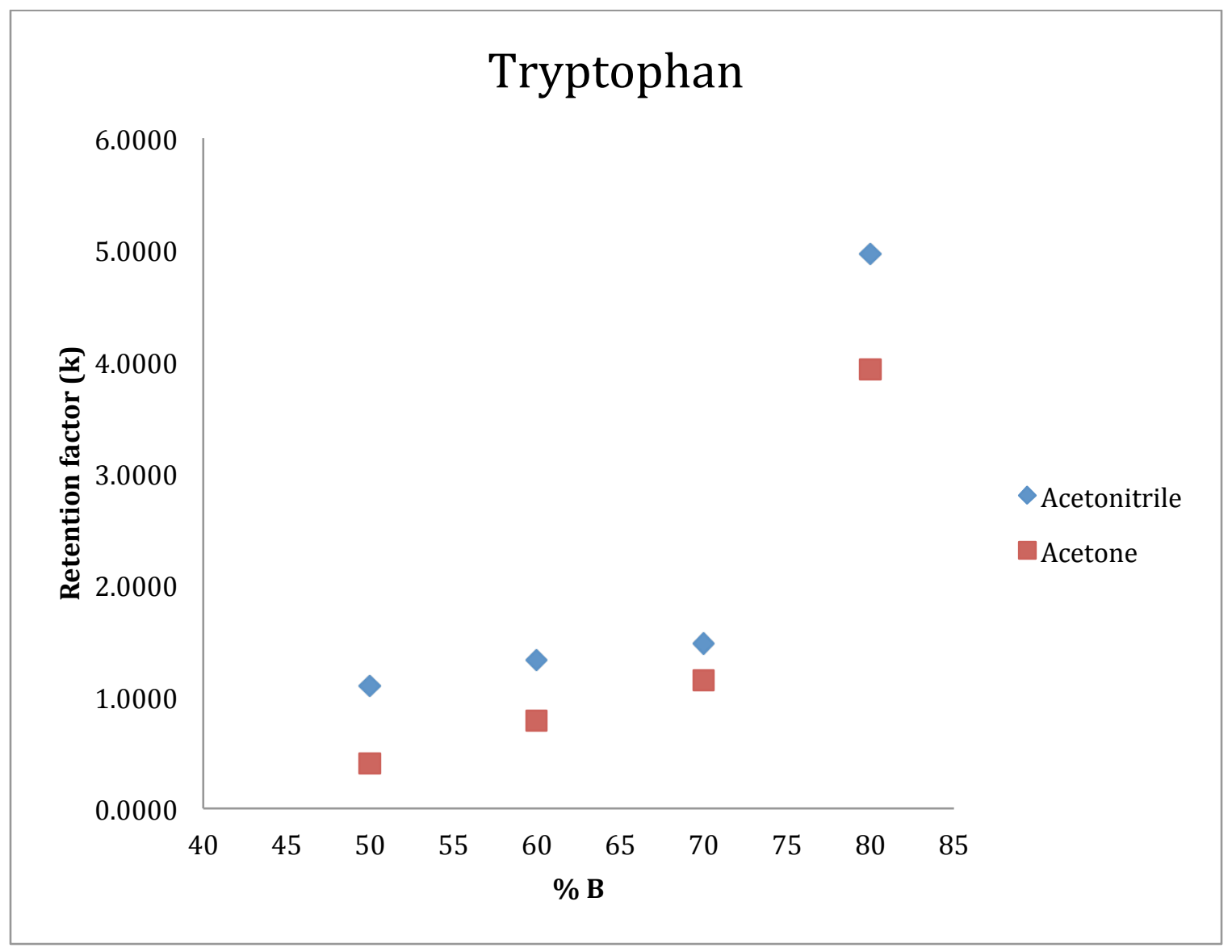

Figure 28: Retention Factor as a Function of \% B for L-tryptophan. 


\section{CONCLUSIONS}

The synthesis procedure of hydrosilation/silanization successfully produced a silica hydride-based stationary phase bonded with $1 \mathrm{H}, 1 \mathrm{H}, 2 \mathrm{H}$ perfluoro - 1 - octene. The reproducibility of the synthetic protocol was established. Successful bonding between the perfluorinated moiety and the silica hydride was qualitatively confirmed by DRIFT, ${ }^{13} \mathrm{C}$ CP-MAS, and elemental analysis.

The carbon elemental analysis provided percent carbon values for the newly synthesized stationary phase. The carbon surface coverage was calculated by the Berendsen and de Galan equation. Low surface coverage by the perfluorinated moiety was achieved in order to study the combined influence of silica hydride and perfluorinated functionality on the retention patterns of test samples.

One of the primary goals of this project was to establish a dual retention profile for polar and nonpolar compounds. Polar compounds were well retained in ANP conditions of higher organic content in the mobile phase. The nonpolar compounds were retained in RP conditions of lower organic content in the mobile phase. ANP and RP data for a series of polar and nonpolar compounds on the perfluorinated stationary phase exhibited a classical U-shaped retention map that is similar to the silica hydride-based stationary phases.

Studying the effect of varying concentrations of the buffer identified a specific trend in the retention time for polar compounds. In this project, varying concentrations of two buffer systems were used: formic acid and ammonium acetate. The compatibility of the ammonium acetate buffer was first tested and confirmed. For polar compounds, with an 
increase in the concentration of buffer (formic acid as well as ammonium acetate) there is a decrease in the retention time. This trend is exactly opposite that observed in the case of hydrophilic interaction chromatography $[15,24]$. Hence, it can be predicted that the retention mechanism involved in silica hydride-based stationary phase is different from HILIC. However, retention times for a neutral compound, toluene, were found to be independent of buffer concentration. This strongly suggests that, in the ANP mode on silica hydride-based stationary phases, the separation mechanism might involve ionic/electrostatic interactions.

Lastly, compatibility of acetone as a nonpolar solvent was tested using ESI LC-MS. Acetone was found to be compatible with silica hydride-based perfluorinated stationary phases and the LC-MS technique. For polar compounds, there was significant retention in ANP conditions. Comparing retention factors for acetone and acetonitrile revealed that acetonitrile is almost $25 \%$ more retentive than acetone.

At this point, a detailed account of the separation mechanism involved in perfluorinated silica hydride-based phases has not been established. Further goals should involve zeta potential measurements for perfluorinated silica hydride stationary phase. The zeta potential of a stationary phase material can be measured within a few minutes with small quantities of mobile phase containing dispersed stationary phase particles. This method is very sensitive to the charge on the particle. Zeta potential measurements would help to study the effects of the mobile phase composition and varying $\mathrm{pH}$ on the ionization of functional groups or water-enriched layers on the stationary phase surface. Zeta potential 
measurements would provide insight into the investigation of separation forces acting in the silica hydride based stationary phase [29].

Overall, the key goals of this project were successfully achieved. Preliminary data on retention pattern, $\mathrm{U}$-shaped retention profile, the effect of varying $\mathrm{pH}$ strength of formic acid and ammonium acetate, and compatibility of acetone as a solvent would be useful for further investigation of the perfluorinated silica hydride-based stationary phase. 


\section{REFERENCES}

1. Ettre, L. S.; Sakodynskii, K. I. Chromatographia, 1993, 35(3-4), 223-231.

2. Skoog, A. D.; Holler, F. J.; Nieman, T. A. Principles of Instrumental Analysis, 5th ed.; Saunders College Publishing: Orlando, Florida, 1998; Chapters 26, 28.

3. Skoog, D.; West, D.; Holler, F. L.; Crouch, S. Fundamentals of analytical chemistry, 8th ed.; Cengage Learning: Boston, Massachusetts, 2006; Chapters 30,32 .

4. Moldoveanu, S., David, V. Essentials in modern HPLC separations; Elsevier: Waltham, Massachusetts, 2013; Chapters 1, 3.

5. Niessen, W. M. A. Liquid chromatography-mass spectrometry; CRC Press: Boca Raton, Florida, 2006; Chapters 1, 2.

6. Snyder, L. R., Kirkland, J. J., Dolan, J. W. Introduction to modern liquid chromatography; John Wiley \& Sons: Hoboken, New Jersey, 2011; Chapter 1.

7. http://imgarcade.com/1/liquid-chromatography-diagram/ (accessed February 2nd, 2015)

8. Watson, J. T., Sparkman, O. D. Introduction to mass spectrometry: instrumentation, applications, and strategies for data interpretation; John Wiley \& Sons: Hoboken, New Jersey, 2007; Chapter 1.

9. Heftmann, E. Chromatography: Fundamentals and applications of chromatography and related differential migration methods-Part B: Applications; Elsevier: Boston, Massachusetts, 2004; Chapters 1, 2.

10. Jal, P. K.; Patel, S.; Mishra, B. K. Talanta, 2004, 62(5), 1005-1028.

11. http://microsolvtech.com/hplc/typec_about.asp (accessed on February 27th, 2015)

12. Pesek, J. J.; Matyska, M. T. Journal of separation science, 2005, 28(15), 18451854.

13. Pesek, J. J.; Matyska, M. T.; Boysen, R. I.; Yang, Y.; Hearn, M. T. TrAC Trends in Analytical Chemistry, 2013, 42, 64-73.

14. Pesek, J. J.; Matyska, M. T.; Fischer, S. M.; Sana, T. R. Journal of Chromatography A, 2008, 1204(1), 48-55. 
15. Pesek, J.; Matyska, M. T. A Comparison of Two Separation Modes: HILIC and Aqueous Normal Phase Chromatography, LCGC 25 (2007): 480-490.

16. Euerby, M. R.; McKeown, A. P.; Petersson, P. Journal of separation science, 2003, 26(3-4), 295-306.

17. Berendsen, G. E.; Pikaart, K. A.; De Galan, L.; Olieman, C. Analytical Chemistry, 1980, 52(12), 1990-1993.

18. Xindu, G.; Carr, P. W. Journal of Chromatography A, 1983, 269, 96-102.

19. 19. Przybyciel, M. Fluorinated HPLC phases-Looking beyond $\mathrm{C} 18$ for reversedphase HPLC, LCGC Europe (2006): 19(1), 19-28.

20. Zhang, W. Journal of Fluorine Chemistry, 2008, 129(10), 910-919.

21. Pesek, J. J.; Matyska, M. T. Journal of liquid chromatography \& related technologies, 2006, 29(7-8), 1105-1124.

22. Duer, M. J. Solid state NMR spectroscopy: principles and applications; John Wiley \& Sons, 2007, Chapter 2.

23. Berendsen, G. E.; Galan, L. D. Journal of Liquid Chromatography, 1978, 1(5), 561-586.

24. Boysen, R. I.; Yang, Y.; Chowdhury, J.; Matyska, M. T.; Pesek, J. J.; Hearn, M. T. Journal of Chromatography A, 2011, 1218(44), 8021-8026.

25. Pesek, J. J.; Matyska, M. T.; Prajapati, K. V. Journal of separation science, 2010, 33(19), 2908-2916.

26. Chu, C. H.; Jonsson, E.; Auvinen, M.; Pesek, J. J.; Sandoval, J. E. Analytical Chemistry, 1993, 65(6), 808-816.

27. Pesek, J. J.; Matyska, M. T. Interface Science, 1997, 5(2-3), 103-117.

28. Berendsen, G. E.; Pikaart, K. A.; De Galan, L.; Olieman, C. Analytical Chemistry, 1980, 52(12), 1990-1993.

29. Kulsing, C.; Yang, Y.; Munera, C.; Tse, C.; Matyska, M. T.; Pesek, J. J.; Hearn, M. T. Analytica chimica acta, 2014, 817, 48-60.

30. Sandoval, J. E.; Pesek, J. J. Analytical Chemistry, 1991, 63(22), 2634-2641. 
